\title{
The Binet-Legendre Metric in Finsler Geometry
}

\author{
VLADIMIR S MATVEEV \\ MARC TROYANOV
}

For every Finsler metric $F$ we associate a Riemannian metric $g_{F}$ (called the BinetLegendre metric). The Riemannian metric $g_{F}$ behaves nicely under conformal deformation of the Finsler metric $F$, which makes it a powerful tool in Finsler geometry. We illustrate that by solving a number of named Finslerian geometric problems. We also generalize and give new and shorter proofs of a number of known results. In particular we answer a question of M Matsumoto about local conformal mapping between two Minkowski spaces, we describe all possible conformal self maps and all self similarities on a Finsler manifold. We also classify all compact conformally flat Finsler manifolds, we solve a conjecture of S Deng and Z Hou on the Berwaldian character of locally symmetric Finsler spaces, and extend a classic result by $\mathrm{HC}$ Wang about the maximal dimension of the isometry groups of Finsler manifolds to manifolds of all dimensions.

Most proofs in this paper go along the following scheme: using the correspondence $F \mapsto g_{F}$ we reduce the Finslerian problem to a similar problem for the BinetLegendre metric, which is easier and is already solved in most cases we consider. The solution of the Riemannian problem provides us with the additional information that helps to solve the initial Finslerian problem.

Our methods apply even in the absence of the strong convexity assumption usually assumed in Finsler geometry. The smoothness hypothesis can also be replaced by a weaker partial smoothness, a notion we introduce in the paper. Our results apply therefore to a vast class of Finsler metrics not usually considered in the Finsler literature.

53C60, 58B20; 53C $35,30 \mathrm{C} 20,53 \mathrm{~A} 30$

\section{Introduction}

In the present paper, a Finsler metric on a smooth manifold $M$ is a continuous function $F: T M \rightarrow[0, \infty)$ such that for every point $x \in M$ the restriction $F_{x}=F_{\mid T_{x} M}$ on the tangent space at $x$ is a Minkowski norm, that is $F_{x}$ is positively homogenous and convex and it vanishes only at $\xi=0$ : 
(a) $F_{x}(\lambda \cdot \xi)=\lambda \cdot F_{x}(\xi)$ for any $\lambda \geq 0$

(b) $F_{x}(\xi+\eta) \leq F_{x}(\xi)+F_{x}(\eta)$

(c) $F_{x}(\xi)=0 \Rightarrow \xi=0$

Observe that $F_{x}$ is a norm in the usual sense if and only if it is symmetric, ie $F_{x}(-\xi)=F_{x}(\xi)$. The Finsler metric is said to be of class $C^{k}$ if the restriction of $F$ to the slit tangent bundle $T M^{0}=T M \backslash$ (the zero section) is a function of class $C^{k}$. Note that it is customary in Finsler geometry to require the Finsler metric to be of class $C^{2}$ and strongly convex, that is the Hessian of the restriction of $F^{2}$ to $T_{x} M \backslash\{0\}$ is assumed to be positive definite for any $x \in M$. However we shall avoid these hypotheses as they exclude from the theory some interesting and important examples.

Our goal in this paper is to solve a number of open problems in Finsler geometry by reducing them to problems in Riemannian Geometry. The method is to associate a natural Riemannian metric $g_{F}$ to a given Finsler metric $F$ on a smooth manifold $M$. We use a construction which comes from classical mechanics and convex geometry: we first define the scalar product $g_{F}^{*}$ on the cotangent space $T_{x}^{*} M$ of a given point to be a normalized $L^{2}$ scalar product of the restrictions of $\theta, \phi \in T_{x}^{*} M$ to the unit ball $\Omega_{x}=\left\{\xi \in T_{x} M \mid F(x, \xi) \leq 1\right\} \subset T_{x} M$, that is

$$
g_{F}^{*}(\theta, \varphi)=\frac{(n+2)}{\lambda\left(\Omega_{x}\right)} \int_{\Omega_{x}}(\theta(\eta) \cdot \varphi(\eta)) d \lambda(\eta) .
$$

where $d \lambda$ is an arbitrary linear volume form on $T_{x} M$ and $\lambda\left(\Omega_{x}\right)$ is the volume of $\Omega_{x}$ with respect to $d \lambda$. It is clear that $g_{F}^{*}$ is a scalar product and that it is independent of the choice of $d \lambda$.

Definition 1.1 The Binet-Legendre metric associated to a Finsler metric $F$ on a smooth manifold $M$ is the Riemannian metric $g_{F}$ dual to the scalar product defined above:

$$
g_{F}(\xi, \eta)=g_{F}^{*}\left(\xi^{b}, \eta^{b}\right)
$$

for any $\xi, \eta \in T M$, where $\xi^{b} \in T_{x}^{*} M$ is defined as $g_{F}^{*}\left(\xi^{b}, \theta\right)=\theta(\xi)$ for all $\theta \in T_{x}^{*} M$.

The motivation for the name Binet-Legendre comes from the fact that the unit ball of $g_{F}^{*}$ in the cotangent space $T_{x}^{*} M$ is the so called Binnet ellipsoid of the convex body $\Omega_{x} \subset T_{x} M$, while the unit ball of $g_{F}^{*}$ in the tangent space $T_{x}^{*} M$ is its Legendre ellipsoid (up to a scaling constant; see Remark 12.2). These ellipsoids have their roots in the 19-th century description of rigid bodies dynamics and have more recently been a subject studied in convex geometry; see for example Milman and Pajor [36]. It seems 
that the Binet-Legendre metric has so far not attracted the attention it deserves in Finsler geometry. It appears under the name osculating Riemannian metric in the paper by P Centore [8] where it is proven that the Hausdorff measure on a Finsler manifold is greater or equal to the Binet-Legendre Riemannian volume form. We did not find any other published work on the Binet-Legendre metric in Finsler geometry, although the idea is probably known to the experts.

The Binet-Legendre metric is one among many possible ways to construct a Riemannian metric on a Finsler manifold; its importance lies in the fact that it satisfies the following natural functorial properties.

Theorem 1.2 The Binet-Legendre metric $g_{F}$ associated to the Finsler manifold $(M, F)$ satisfies the following properties:

(a) If $F$ is of class $C^{k}$, then so is $g_{F}$.

(b) If $F$ is Riemannian, ie, if $F(x, \xi)=\sqrt{g_{x}(\xi, \xi)}$ for some Riemannian metric $g$, then $g_{F}=g$.

(c) If $A \in \operatorname{Aut}(T M)$ is a $C^{k}$-field of automorphisms of the tangent bundle of $M$, then $g_{A^{*} F}=A^{*} g_{F}$.

(d) If $F_{1}(x, \xi)=\lambda(x) \cdot F_{2}(x, \xi)$ for some function $\lambda: M \rightarrow \mathbb{R}_{+}$, then $g_{F_{1}}=\lambda^{2} \cdot g_{F_{2}}$.

(e) If $\frac{1}{\lambda} \cdot F_{1} \leq F_{2} \leq \lambda \cdot F_{1}$ for some function $\lambda: M \rightarrow \mathbb{R}_{+}$, then

$$
\frac{1}{\lambda^{2 n}} \cdot g_{F_{1}} \leq g_{F_{2}} \leq \lambda^{2 n} \cdot g_{F_{1}} \text {. }
$$

Proof The first property is Theorem 2.4, which is actually a stronger result, combined with example (a) in Section 2 below. Properties (b)-(e) are essentially known facts about the Legendre ellipsoid. For the convenience of the reader we prove them in the Appendix; see Proposition 12.1.

This theorem immediately implies the following consequences:

- If we have that two Finsler metrics $F_{1}$ and $F_{2}$ are conformally equivalent, ie, if $F_{1}(x, \xi)=\lambda(x) \cdot F_{2}(x, \xi)$ for some function $\lambda: M \rightarrow \mathbb{R}$, then the corresponding Riemannian metrics are also conformally equivalent with essentially the same conformal factor: $g_{F_{1}}=\lambda^{2} \cdot g_{F_{2}}$. In particular every conformal transformation of the Finsler metrics is conformal for the Binet-Legendre metric.

- The Binet-Legendre construction is $C^{0}$-stable: if $F_{1}$ and $F_{2}$ are $C^{0}$-close, then so are $g_{F_{1}}$ and $g_{F_{2}}$.

- If $F_{1}$ and $F_{2}$ are bilipschitzly equivalent, then so are $g_{F_{1}}$ and $g_{F_{2}}$. 
Note that besides the Binet-Legendre metric, we could use other procedures that associate a scalar product (or an ellipsoid) to a given convex body, such as the one based on the John or Löwner ellipsoid, or the constructions by Lutwak, Yang, and Zhang [30; 31], the authors, Rademacher and Zeghib [35], and Szabó [44; 43]. It is however not completely clear whether the above properties, in particular the smoothness or $C^{0}$-stability, still hold for those alternative constructions.

We shall give concrete applications of the Binet-Legendre metric to the solutions of the following seven geometric problems:

(1) A generalization of the result of Wang [47] about the possible dimensions of the isometry groups of Finsler manifolds to manifolds of all dimensions.

(2) The description of local conformal maps between Minkowski spaces, thus answering a question raised by Matsumoto in [32].

(3) The description of all Finsler spaces admitting a nontrivial self-similarity.

(4) The topological classification of conformally flat compact Finsler manifolds.

(5) The description of all conformal self maps in a Finsler manifold.

(6) A short proof of a theorem of Szabó on Berwald spaces.

(7) A positive solution to the conjecture of S Deng and Z Hou [14] stating that a locally symmetric Finsler space is Berwald.

Most of these problems are related to conformal or isometric mappings of Finsler metrics. Then, the Binet-Legendre metric enjoys the same geometric condition as the given Finsler metric. In most problems we consider (the exceptions are Problem (4) and, to a certain extent, Problem (3)), the Riemannian analog of the problem is well-studied or can be easily described. This gives us additional information about the geometry of the manifold that we use in the solutions of the above mentioned problems. There is no general schematic way one can use this additional Riemannian information; in certain cases it is straightforward and in certain cases it is tricky.

An additional result of our paper is the construction of a family of new scalar invariants of Finsler manifolds extending the well known Minkowski functionals from convex geometry. The invariants can be effectively calculated numerically and one can use them to decide whether two explicitly given Finsler metrics are conformally equivalent or isometric.

The paper is organized as follows. In Section 2 we introduce partially smooth Finsler metrics and show the corresponding Binet-Legendre metrics are smooth. Sections 3-9 are devoted to the solutions of the aforementioned geometric problems. In Section 10 
we use the Binet-Legendre metric to produce new conformal invariants of Finsler metrics. In the Appendix, we rapidly prove the basic properties of the Binet-Legendre construction.

Acknowledgements This work benefited from discussions with J C Álvarez Paiva, D Burago, S Deng, S Ivanov, A Petrunin, R Schneider, Z Shen and J Szilasi. We also thank the Swiss National Science Foundation (200020-130107) and the Deutsche Forschungsgemeinschaft (GK 1523) for their financial support as well EPF Lausanne and FSU Jena for their hospitality.

\section{Partially smooth Finsler metrics}

There are very natural examples of Finsler metrics which are not smooth in the usual sense. In this section we discuss a notion of smoothness that is weaker than the one usually considered in Finsler geometry and yet is still amenable to the techniques of differential geometry via the use of the Binet-Legendre metric. A homogeneous diffeomorphism of a finite-dimensional vector space $V$ is a diffeomorphism $A: V \backslash\{0\} \rightarrow V \backslash\{0\}$ such that for every $\lambda>0$ and for every $v \in V, v \neq 0$ we have $A(\lambda v)=\lambda A(v)$.

A field of homogeneous diffeomorphisms of $T M$ is a diffeomorphism $A: T M^{0} \rightarrow T M^{0}$, where $T M^{0}=T M \backslash$ (the zero section) such the restriction $A_{x}=A_{\mid T_{x} M}$ is a homogeneous diffeomorphism of the tangent space $T_{x} M$ for every $x \in M$.

Definition 2.1 Let $(M, F)$ be a Finsler manifold and $U \subseteq M$ the domain of some coordinate system $\left(x_{1}, \ldots, x_{n}\right)$. Then $F$ is said to be $C^{k}$-partially smooth in the coordinates $x_{i}$ if there exists a $C^{k}$-smooth field of homogeneous diffeomorphisms $A: T U^{0} \rightarrow T U^{0}$ such that the function $x \mapsto F\left(x, A_{x}(\xi)\right)$ is of class $C^{k}$ in $U$ for any fixed $\xi \in \mathbb{R}^{n}$.

In this definition, we use the identification $T U=U \times \mathbb{R}^{n}$ defined by the coordinate system. The vector field $\xi$ is thus "constant in the coordinate system $x_{i}$ ".

Lemma 2.2 Let $U$ be a domain in a Finsler manifold $(M, F)$. If $F$ is $C^{k}$-partially smooth in some coordinate system on $U$, then it is partially smooth in any coordinate system on $U$.

Partial smoothness in some coordinate domain is thus in fact an intrinsic notion, and we are led to the following global definition.

Definition 2.3 A Finsler manifold is $C^{k}$-partially smooth if it is $C^{k}$-partially smooth in some neighborhood of any of its point. 
Proof of Lemma 2.2 From the local nature of the concept, on may assume that $M$ is a domain $U \subset \mathbb{R}^{n}$ and that the Finsler metric $F$ is $C^{k}$-partially smooth in the usual coordinates of $\mathbb{R}^{n}$. We consider a field $A$ of homogeneous diffeomorphisms as in Definition 2.1: $A$ is $C^{k}$-smooth and the mapping $x \mapsto F\left(x, A_{x}(\xi)\right)$ is of class $C^{k}$ in $U$ for any constant vector field $\xi$. Let $y_{j}$ be another coordinate system on $U$, specifically, let $\phi: V \rightarrow U$ be a diffeomorphism from some domain $V$ onto $U$ and set $x=\phi(y)$. The Finsler structure $F$ on $U$ transforms into the Finsler structure $\widetilde{F}$ on $V$ defined as

$$
\widetilde{F}(y, \xi)=F\left(\phi(y), d \phi_{y}(\xi)\right) .
$$

Define now the field of homogeneous diffeomorphism $\tilde{A}$ as $\tilde{A}_{y}=d \phi_{y}^{-1} \circ A_{\phi(y)}$. For any fixed vector $\xi \in \mathbb{R}^{n}$, the function

$$
V \ni y \mapsto \widetilde{F}\left(y, \widetilde{A}_{y}(\xi)\right)=F\left(\phi(y), d \phi_{y} \circ \widetilde{A}_{y}(\xi)\right)=F\left(\phi(y), A_{\phi(y)}(\xi)\right)
$$

is the composition of the $C^{k}$ functions $\phi: V \rightarrow U$ and $x \mapsto F\left(x, A_{x}(\xi)\right)$, therefore

$$
y \mapsto \tilde{F}\left(y, \tilde{A}_{y}(\xi)\right)
$$

is of class $C^{k}$ for any constant vector $\xi$ and we conclude that $\widetilde{F}$ is partially smooth in the coordinates $y_{j}$.

Let us give some examples of partially smooth Finsler metrics.

(a) Every smooth Finsler metric is partially smooth.

(b) A Minkowski Finsler metric $F(\xi)$ on $\mathbb{R}^{n}$ is partially smooth. Indeed, we canonically identify $T \mathbb{R}^{n}$ with $\mathbb{R}^{n} \times \mathbb{R}^{n}$ and look at $F$ as a "function of 2 variables which is constant in the first variable": $F(x, \xi)=F(\xi)$, ie, the field $A$ of the homogeneous diffeomorphisms consists of identities $\mathrm{id}_{x}: T_{x} M \rightarrow T_{x} M$.

(c) Let $F_{1}$ and $F_{2}$ be Finsler metrics on the same manifold such that $F_{1}$ is partially smooth and $F_{2}$ is smooth. Let $h_{1}, h_{2}: M \rightarrow[0, \infty)$ be smooth nonnegative functions on $M$ such that $h_{1}(x)+h_{2}(x)>0$ for all $x \in M$. Then, the Finsler metric

$$
F(x, \xi)=h_{1}(x) F_{1}(x, \xi)+h_{2}(x) F_{2}(x, \xi)
$$

is again a partially smooth Finsler metric.

(d) As a special case of the previous example, consider the Finsler metric on $M=\mathbb{R}^{2}$ given by

$$
F\left(x_{1}, x_{2}, \xi_{1}, \xi_{2}\right)=\left(1-f\left(x_{1}\right)\right) \cdot\left(\left|\xi_{1}\right|+\left|\xi_{2}\right|\right)+f\left(x_{1}\right) \cdot \sqrt{\xi_{1}^{2}+\xi_{2}^{2}},
$$

where $f: \mathbb{R} \rightarrow[0,1]$ is a smooth function such that $f(x)=0$ for $x \leq-1$, $f(0)=1 / 2$ and $f(x)=1$ for $x \geq 1$. The Finsler metric $F$ is partially smooth, 
it is independent of the variable $x_{2}$ and it interpolates from the $L^{1}$ norm on the plane to the Euclidean $\left(L^{2}\right)$ norm as $x_{1}$ varies from -1 to 1 .

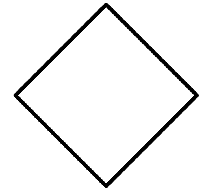

Unit ball for $x_{1} \leq-1$

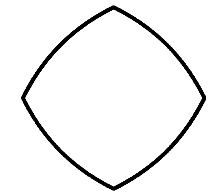

Unit ball for $x_{1}=0$

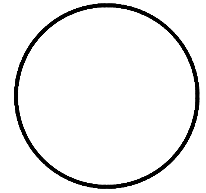

Unit ball for $x_{1} \geq 1$

Figure 1: The unit balls in example (d) for different values of $x_{1}$.

(e) Let $F$ be a partially smooth metric on $M$. Consider a field $A_{x}: T_{x} M \rightarrow T_{x} M$ of invertible endomorphisms of the tangent bundle (ie $A$ is an invertible $(1,1)$ tensor field), and the new Finsler structure defined by $F_{A}(x, \xi)=F\left(x, A_{x}(\xi)\right)$. (Observe that any Riemannian metric on a domain in $\mathbb{R}^{n}$ can be obtained from the Euclidean metric by this procedure.) Then, this metric is partially smooth.

(f) Consider smooth functions $f_{1}, \ldots, f_{n}: \mathbb{R}^{2} \rightarrow \mathbb{R}^{2}$ such that for every $x \in \mathbb{R}^{2}$ the points $f_{1}(x), \ldots, f_{n}(x)$ are the vertices of a convex polygon $P_{x}$ such that the point 0 lies in its interior. We identify $T \mathbb{R}^{2}$ with $\mathbb{R}^{2} \times \mathbb{R}^{2}$ and consider the Finsler metric whose $\Omega_{x}=P_{x}$ at every $x \in \mathbb{R}^{2}$. Then, this metric is partially smooth.

The latter example was in fact one of our original motivations for introducing the notion of partially smooth Finsler metric. This example also suggests the following remark: Finsler geometry can be used to describe certain phenomena in natural sciences (such as light prolongation in crystals or certain diffusion processes in organic cells), but to use Finsler geometry in such context, one needs to accept nonsmooth metrics and the class of partially smooth Finsler metrics seems quite appropriate. Indeed, the cells or crystals can be viewed as a field of convex bodies at every point of $\mathbb{R}^{3}$ or of $\mathbb{R}^{2}$ and can be described by a Finsler metric. In particular the Finsler metric in example (f) above could be relevant in describing crystal structures.

The notion of partially smooth Finsler metrics is mainly motivated by the following result.

Theorem 2.4 The Binet-Legendre metric of a $C^{k}$-partially smooth Finsler manifold is a Riemannian metric of class $C^{k}$. 
Proof Let $U \subseteq M$ be the domain of some coordinate system $x_{1}, \ldots, x_{n}$. We first prove that the function $x \mapsto \operatorname{Vol}\left(\Omega_{x}\right)$ is of class $C^{k}$ in $U$ where $\Omega_{x} \subseteq T_{x} U=\mathbb{R}^{n}$ the Finsler unit ball and $\operatorname{Vol}\left(\Omega_{x}\right)$ is its Euclidean volume.

By hypothesis, there is a $C^{k}$ field of homogeneous diffeomorphisms $A: T U^{0} \rightarrow T U^{0}$ such that $x \mapsto F\left(x, A_{x}(\xi)\right)$ is of class $C^{k}$ for any fixed $\xi \in \mathbb{R}^{n}$. Let us define $\Omega_{x}^{\prime}=A_{x}^{-1}\left(\Omega_{x}\right)$. Writing $\xi^{\prime}=A_{x}(\xi)$, we have

$$
\begin{gathered}
\Omega_{x}^{\prime}=\left\{\xi^{\prime} \in \mathbb{R}^{n} \mid F\left(x, A_{x}\left(\xi^{\prime}\right)\right)<1\right\}, \\
\operatorname{Vol}\left(\Omega_{x}\right)=\int_{\Omega_{x}} d \xi=\int_{F\left(x, A_{x}\left(\xi^{\prime}\right)\right)<1} \operatorname{Jac}\left(A_{x}\right)\left(\xi^{\prime}\right) d \xi^{\prime} .
\end{gathered}
$$

Using polar coordinates $\xi^{\prime}=r \cdot u$, with $u \in S^{n-1}$, this gives

$$
\operatorname{Vol}\left(\Omega_{x}\right)=\int_{S^{n-1}}\left(\int_{r=0}^{1 / F(x, A(u))} \operatorname{Jac}\left(A_{x}\right)(r \cdot u) r^{n-1} d r\right) d u,
$$

where $d u$ stands for the spherical measure on $S^{n-1}$ and $\operatorname{Jac}\left(A_{x}\right)$ is the Jacobian determinant $\operatorname{det}\left(\partial \xi / \partial \xi^{\prime}\right)$. Since the functions $\operatorname{Jac}\left(A_{x}\right)$ and the bound $1 / F(x, A(u))$ $C^{k}$ - smoothly depend on $x$, the integral

$$
I(x, u)=\int_{r=0}^{1 / F(x, A(u))} \operatorname{Jac}\left(A_{x}\right)(r \cdot u) r^{n-1} d r
$$

also smoothly depends on $x$. Then,

$$
\operatorname{Vol}\left(\Omega_{x}\right)=\int_{S^{n-1}} I(x, u) d u
$$

smoothly depends on $x$ as we claimed.

The proof for the Binet-Legendre metric is similar. It suffices to prove that the dual metric $g^{*} F$ is smooth, ie that $x \mapsto\left(g_{F}\right)_{x}^{*}(\theta, \theta)$ is smooth in $U$ for any fixed covector $\theta: \mathbb{R}^{n} \rightarrow \mathbb{R}$. We denote by $\Theta(\xi)$ the function $\theta(\xi)^{2}$ and by $\widetilde{\Theta}$ the function $\Theta \circ A_{x}$. Arguing as above and using (1), we have

$$
\begin{aligned}
\frac{\operatorname{Vol}\left(\Omega_{x}\right)}{(n+2)} \cdot g_{F_{x}}^{*}(\theta, \theta) & =\int_{\Omega_{x}} \Theta(\xi) d \xi=\int_{F\left(x, A_{x}\left(\xi^{\prime}\right)\right)<1} \Theta\left(A_{x}(\xi)\right) \operatorname{Jac}\left(A_{x}\right)\left(\xi^{\prime}\right) d \xi^{\prime} \\
& =\int_{S^{n-1}}\left(\int_{r=0}^{1 / F(x, A(u))} \tilde{\Theta}(r \cdot u) \operatorname{Jac}\left(A_{x}\right)(r \cdot u) r^{n-1} d r\right) d u
\end{aligned}
$$

This is again a $C^{k}$ function of $x \in U$, which completes the proof. 


\section{On the number of Killing vector fields}

By Theorem 1.2(d), the group of isometries of a partially smooth Finsler manifold $(M, F)$ is a subgroup of the group of isometries of $\left(M, g_{F}\right)$. It is a closed subgroup and therefore it is a Lie group and its dimension is at most $\frac{1}{2} n(n+1)$; for smooth strongly convex Finsler metrics this statement is known; see Deng and Hou [12, Theorem 3.3].

In 1947, HC Wang proved that a smooth and strongly convex $n$-dimensional Finsler manifold of dimension $n \neq 2,4$ is Riemannian if its group of isometries has dimension greater than $\frac{n(n-1)}{2}+1$; see [47] and Yano [48]. Our next result extends Wang's theorem to all dimensions. Our proof is more direct and also works for partially smooth metrics and without the strong convexity condition. This theorem gives a positive answer to a question raised by S Deng and Z Hou in [13, page 660].

A vector field $K$ on a Finsler manifold $(M, F)$ is said to be a Killing vector field if it generates a local flow $\phi_{t}^{k}$ of local isometries for the metric $F$.

Theorem 3.1 Let $\left(M^{n}, F\right)$ be a partially $C^{2}$-smooth connected Finsler manifold. If the dimension of the space of Killing vector fields of $(M, F)$ is greater than $\frac{n(n-1)}{2}+1$, then $F$ is actually a Riemannian metric.

Observe that the bound given in the theorem is sharp: The (non-Riemannian) Minkowski space $\mathbb{R}^{n}$ with smooth and strongly convex norm

$$
F(\xi)=\left(\left(\sum_{i=1}^{n} \xi_{i}^{2}\right)^{2}+\xi_{n}^{4}\right)^{1 / 4}
$$

has $r=n+\operatorname{dim} S O(n-1)=\frac{n(n-1)}{2}+1$ linearly independent complete Killing vector fields.

Proof Let $r>\frac{n(n-1)}{2}+1$ be the dimension of the space of Killing vector fields. Take a point $x$ and choose $r-n$ linearly independent Killing vector fields $K_{1}, \ldots, K_{r-n}$ vanishing at $x$, this is possible because the dimension $T_{x} M$ is $n$. The point $x$ is then a fixed point of the corresponding local flows $\phi_{t}^{K_{1}}, \ldots, \phi_{t}^{K_{r-n}}$. It is obvious that any Killing vector field for $F$ is also a Killing vector field of $g_{F}$. In particular, for every fixed $t$, the differentials of $\phi_{t}^{K_{1}}, \ldots, \phi_{t}^{K_{r-n}}$ at $x$ are linear isometries of $\left(T_{x} M, g_{F}\right)$. Let us denote by $\Phi_{i} \in \operatorname{End}\left(T_{x} M\right)$ the differentials $\Phi_{i}=\left(d / d t\left(d_{x} \phi_{t}^{K_{i}}\right)\right)_{\mid t=0}$. We claim $\Phi_{1}, \ldots, \Phi_{r-n}$ are linearly independent. Indeed, assume $\sum_{i=1}^{r-n} a_{i} \Phi_{i}=0$ for some constants $a_{i} \in \mathbb{R}$ and consider the Killing field $K=\sum_{i=1}^{r-n} a_{i} K_{i}$. Let us denote 
by $\phi_{t}^{K}$ the (local) flow generated by $K$; because $\phi_{t}^{K} \circ \exp _{x}=\exp _{x} \circ d_{x} \phi_{t}^{K}$, we have for $y=\exp _{x}(\xi)$

$$
K_{y}=\left.\frac{d}{d t}\right|_{t=0} \phi_{t}^{k}(y)=\left.\frac{d}{d t}\right|_{t=0} \exp _{x}\left(d \phi_{t}^{K}(\xi)\right)=0,
$$

since $\left(d / d t\left(d_{x} \phi_{t}^{K}\right)\right)_{\mid t=0}=\sum_{i=1}^{r-n} a_{i} \Phi_{i}=0$. It follows that $K=0$ in an open neighborhood of the point $x$ implying $K \equiv 0$ on the whole manifold. Because $K_{i}$ are assumed to be linearly independent, we have $a_{i}=0$ for all $i$ and $\Phi_{i}$ are thus linearly independent as claimed.

We now denote by $G \subset S O\left(T_{x} M, g_{F}\right)$ the smallest closed subgroup of $S O\left(T_{x} M, g_{F}\right)$ generated by the differentials of $\phi_{t}^{K_{1}}, \ldots, \phi_{t}^{K_{r-n}}$ at $x$. Its Lie algebra contains the linearly independent elements $\Phi_{1}, \ldots, \Phi_{r-n}$ and we thus have $\operatorname{dim}(G) \geq r-n$. It is known that for every $n \geq 2$, any $(r-n)$-dimensional subgroup of the orthogonal group $S O(n) \cong S O\left(T_{x} M, g_{F}\right)$ acts transitively on the $g_{F}$ unit sphere $S^{n-1} \subset T_{x} M$ provided $r>\frac{1}{2} n(n-1)+1$. Indeed, for $n \neq 4$, this immediately follows for the classical result of Montgomery and Samelson [37]: they proved that for $n \neq 4$, there exists no proper subgroup of $S O(n)$ of dimension greater than $(n-1)(n-2) / 2$. In dimension 4 , the transitivity follows for example of Ishihara [21, Section 1], where all Lie subgroups of $S O(4)$ are described.

Since the action of $G$ on $T_{x} M$ preserves $F$ and $g_{F}$ and $G$ acts transitively on the $g_{F}$-sphere $S^{n-1} \subset T_{x} M$, the ratio $F(\xi)^{2} / g(\xi, \xi)$ is constant for all $\xi \in T_{x} M^{0}$ implying that $F(\xi)=\lambda(x) \cdot \sqrt{g_{F}(\xi, \xi)}$ for some function $\lambda: M \rightarrow \mathbb{R}_{+}$and for all $\xi \in T M$. This proves that $F$ is Riemannian, furthermore, by Theorem 1.2(b), the coefficient $\lambda \equiv 1$ so that $g_{F}$ coincides with $F$ in the sense that $g_{F}(\xi, \xi)=F^{2}(\xi)$ for all $\xi \in T M$.

Observe that hypothesis of $C^{2}$ partial smoothness of the metric was not really used in the proof, we only used that the flows of the Killing vector fields are of class $C^{1}$, which is automatically fulfilled if the metric is $C^{2}$-partially smooth.

Remark 3.2 Smooth Riemannian manifolds with large groups of isometries have been studied thoroughly; see eg Kobayashi and Nagano [23] for a survey of classical results. In particular, connected Riemannian manifolds with more than $\frac{1}{2} n(n-1)+1$ Killing vector fields are classified as follows. Let $r$ be the dimension of the space of Killing vector fields. Then we have:

(1) If $r>\frac{n(n-1)}{2}+1$ and $n \neq 4$, then $g$ has constant sectional curvature (see [48]).

(2) If $n=4$ and $r>\frac{1}{2} n(n-1)+2=8$, then $g$ also has constant sectional curvature (see [21]). 
(3) If $n=4$ and $r>\frac{1}{2} n(n-1)+1=7$ then either $M$ is Kählerian with constant holomorphic sectional curvature (in this case, $r=8$ ), or $M$ has constant sectional curvature (see [21, Theorem A', page 364]).

Note that although the cited references assume the Killing vector fields to be complete, the proofs work without this hypothesis. Note also a Riemannian manifold with constant sectional curvature locally has $\frac{n(n+1)}{2}$ linearly independent Killing fields.

\section{The Liouville Theorem for Minkowski spaces and the solu- tion to a problem by Matsumoto}

A famous theorem by Joseph Liouville states that any conformal transformation of a domain in $\mathbb{R}^{3}$ to another such domain is either the restriction of a similarity or the composition of an isometry with an inversion, it is, in other words, the restriction of a Möbius transformation. This result was announced in 1850 by Liouville in [28], and the proof appeared as a note in the fifth edition of Monge's book Application de l'analyse à la géométrie [27]. It is well known that this theorem also holds in $\mathbb{R}^{n}$ for $n \geq 3$. By contrast, in dimension 2 the Cauchy-Riemann equations imply that a transformation is conformal if and only if it is either holomorphic or antiholomorphic.

Our next statement says that Liouville's Theorem still holds in non-Euclidean Minkowski spaces. We have in fact a stronger result.

Theorem 4.1 Let $\left(V_{1}, F_{1}\right)$ and $\left(V_{2}, F_{2}\right)$ be two non-Euclidean Minkowski spaces of the same dimension $n \geq 2$. If $f: U_{1} \rightarrow U_{2}$ is a conformal map between two domains $U_{1} \subset V_{1}$ and $U_{2} \subset V_{2}$, then $\left(V_{1}, F_{1}\right)$ and $\left(V_{2}, F_{2}\right)$ are isometric and $f$ is (the restriction of) a similarity, that is the composition of an isometry and a homothety $x \mapsto$ const $\cdot x$.

Remark In the last sentence of the paper [32], M Matsumoto asked whether there exist two locally Minkowski spaces which are conformal to each other. The above theorem shows that the answer to this question is negative unless the metrics are Euclidean or the conformal correspondence is a similarity.

Proof We will first prove the theorem for $n \geq 3$. Fix a point $x \in U_{1} \subseteq V_{1}$ and let $y=f(x) \in U_{2} \subseteq V_{2}$ be the image point. Because $f$ is a conformal map, we have $d f_{x}^{*}\left(F_{2}\right)=\lambda(x) F_{1}$ for some function $\lambda(x)>0$, hence the map $\frac{1}{\lambda(x)} \cdot d f_{x}$ is an isometry from $\left(T_{x} V_{1}, F_{1}\right)$ to $\left(T_{y} V_{2}, F_{2}\right)$, but since a Minkowski space is isometric to its tangent space at any point it follows that $\left(V_{1}, F_{1}\right)$ and $\left(V_{2}, F_{2}\right)$ are isometric. 
From now on, we assume $V_{1}=V_{2}=\mathbb{R}^{n}$ and $F_{1}=F_{2}=F$ is an arbitrary nonEuclidean Minkowski norm. Changing coordinates if necessary, one may also assume the Binet-Legendre scalar product $g_{F}$ of $F$ is the standard scalar product $\langle\cdot, \cdot\rangle$ of $\mathbb{R}^{n}$. It follows $f$ is a conformal map in the usual sense between two domains $U, V \subseteq \mathbb{R}^{n}$.

By the classical Liouville Theorem, $f$ is the restriction of a Möbius transformation, and such a map is known to be either a similarity or the composition of an isometry and an inversion. We thus only need to prove the composition of an isometry and an inversion cannot be a conformal map of some non-Euclidean Minkowski norm F on $\mathbb{R}^{n}$. We now prove the last assertion by contradiction. The map $f$ is of the type

$$
f(x)=Q\left(r^{2} \cdot \frac{x-c}{|x-c|^{2}}\right)+b,
$$

where $r>0$ and $Q$ is a linear orthogonal transformation. The differential of $f$ at a point $x$ is then

$$
d f_{x}(\xi)=r^{2} Q\left(\frac{|x-c|^{2} \cdot \xi-2\langle x-c, \xi\rangle \cdot(x-c)}{|x-c|^{4}}\right) .
$$

Observe that if $x=c+r \cdot v$ with $|v|=1$, then $d f_{x}=Q \circ R_{v}$ where $R_{v}$ is the reflection across the hyperplane $v^{\perp}$. In particular $d f_{x}$ is an isometry for the Euclidean norm. Now since $f$ is a conformal map and the Binet-Legendre scalar product coincides with the standard scalar product on $\mathbb{R}^{n}$, Proposition 12.1 (c) implies that $d f_{x}$ is also an isometry for $F$, that is $F\left(Q \circ R_{v}(\xi)\right)=F(\xi)$ for every $\xi$ and every unit vector $v$. Since the mappings of the form $\xi \mapsto Q\left(R_{v}(\xi)\right)$, where $v \in S^{n-1}$, generate the orthogonal group, our Minkowski norm $F$ is $O(n)$-invariant and is therefore Euclidean. The theorem is proved for $n \geq 3$.

Let us now prove it for $n=2$. We again consider $\mathbb{R}^{2}$ with a fixed Minkowski metric which we denote by $F$, and assume that $g_{F}$ is the standard flat metric. Let us use the conformal structure to construct a family of parallel lines on $\mathbb{R}^{2}$. Take a point $x$ and consider the unit circle $S_{x}^{1} \subset T_{x} \mathbb{R}^{2}$ in the metric $g_{F}$. We take a connected component $I_{\max }^{0}$ of the "maximal" set

$$
I_{\max }=\left\{\xi \in S_{1}(x) \mid F(\xi)=\max _{\eta \in S_{x}^{1}} F(\eta)\right\} .
$$

The set $I_{\max }^{0}$ cannot coincide with the whole $S_{1}(x)$ and is therefore a connected interval. Let $\xi \in S_{1}(x)$ be its midpoint (with respect to the metric on $S_{x}^{1}$ induced by $g_{F}$ ).

The vector $\xi$ is not always unique ( $I_{\max }$ can have more than one connected component, and every connected component has its own midpoint). We choose one of them. 
Note that the construction of the vector $\xi$ is conformally invariant in the following sense: if we multiply $F$ at a point $x$ by a number $\lambda$, the vector $\xi$ is divided by $\lambda$, so the direction of this vector field remains the same.

Now let us extend the vector to all points of $\mathbb{R}^{2}$ by parallel translations, thus obtaining a vector field that we denote by $\xi$. Let $f: U_{1} \rightarrow U_{2}$ be a conformal (ie holomorphic or antiholomorphic) mapping. Then, it sends the vector field $\xi$ to another vector field $\xi^{\prime}=f_{*}(\xi)$ that satisfies the following properties by construction:

(1) $\xi^{\prime}$ is a smooth vector field.

(2) At every point, $\xi^{\prime}$ is the mid vector of a connected component of $I_{\max }$.

Therefore the integral curves of $\xi^{\prime}$ are parallel lines in $\mathbb{R}^{2}$. It is well known (and easy to check) that a holomorphic or antiholomorphic map that sends a family of parallel lines to a family of parallel lines is of the type $f(z)=a z+b$ or $f(z)=a \bar{z}+b$ with $a, b \in \mathbb{C}, a \neq 0$. Thus $f$ is a similarity and the proof is complete.

\section{Conformally flat compact Finsler Manifolds}

A Finsler manifold $(M, F)$ is conformally flat, if there is an atlas whose changes of coordinates are conformal diffeomorphisms between open sets in some Minkowski space. Assuming $M$ to be non-Riemannian, it follows from Theorem 4.1, that these changes of coordinates are Euclidean similarities. The manifold $M$ carries therefore a similarity structure. It turns out that compact manifolds with a similarity structure have been topologically classified by N H Kuiper and D Fried: They are either Bieberbach manifolds (ie $\mathbb{R}^{n} / \Gamma$, where $\Gamma$ is some crystallographic group of $\mathbb{R}^{n}$ ), or they are Hopf manifolds, ie compact quotients of $\mathbb{R}^{n} \backslash\{0\}=S^{n-1} \times \mathbb{R}_{+}$by a group $G$ which is a semidirect product of an infinite cyclic group with a finite subgroup of $O(n+1)$; see $[17 ; 24]$ and Vaisman and Reischer [45]. We thus conclude the following.

Theorem 5.1 A partially smooth connected compact conformally flat non-Riemannian Finsler manifold is either a Bieberbach manifold or a Hopf manifold. In particular, it is finitely covered either by a torus $T^{n}$ or by $S^{n-1} \times S^{1}$.

The structure of Riemannian conformally flat manifold is more complicated; see the discussions by Kulkarni in [25], Matsumoto in [33] and Schoen and Yau in [41]. 


\section{Finsler spaces with a nontrivial self-similarity}

The next theorem concerns forward complete Finsler manifolds. Recall that the distance $d(x, y)$ between two points $x$ and $y$ on a Finsler manifold $(M, F)$ is the infimum of the length

$$
L_{F}(\gamma)=\int_{0}^{1} F(\gamma(t), \dot{\gamma}(t)) d t
$$

of all smooth curves $\gamma:[0,1] \rightarrow M$ joining these two points (ie, $\gamma(0)=x, \gamma(1)=y$ ). This distance satisfies the axioms of a metric except perhaps the symmetry, ie the condition $d(x, y)=d(y, x)$ is usually not satisfied. Together with the distance comes the notion of completeness: the Finsler manifold $(M, F)$ is said to be forward complete if every forward Cauchy sequence converges. A sequence $\left\{x_{i}\right\} \subseteq M$ is forward Cauchy if for any $\varepsilon>0$, there exists an integer $N$ such that $d\left(x_{i}, x_{i+k}\right)<\varepsilon$ for any $i \geq N$ and $k \geq 0$.

A $C^{1}-$ map $f:(M, F) \rightarrow\left(M^{\prime}, F^{\prime}\right)$ is a similarity if there exists a constant $a>0$ (called the dilation constant) such that $F\left(f(x), d f_{x}(\xi)\right)=a \cdot F(x, \xi)$ for all $(x, \xi) \in T M$. It is an isometry if $a=1$.

Clearly a similarity satisfies $d_{F^{\prime}}(f(x), f(y))=a \cdot d_{F}(x, y)$ for all $x, y \in M$ and it follows from the Busemann-Mayer Theorem that any $C^{1}$-map satisfying this condition is a similarity in the previous sense.

Theorem 6.1 Let $(M, F)$ be a forward complete connected $C^{0}$-Finsler manifold. If there exists a nonisometric self-similarity $f: M \rightarrow M$ of class $C^{1}$, then $(M, F)$ is a Minkowski space, that is it is isometric to any one of its tangent spaces.

The proof below is based on a blow up argument familiar in metric geometry and requires no smoothness of the Finsler metric.

Proof We first show that the map $f$ is a bijection. The injectivity follows from the fact that $d(f(x), f(y))=a \cdot d(x, y)$, for any $x, y$ and $a>0$. To show that $f$ is surjective, we observe that $f(M) \subset M$ is open since $f$ is an immersion and $f(M) \subset M$ is closed since it is a forward complete set. Hence $f(M)=M$ and $f$ is thus bijective.

Replacing $f$ by $f^{-1}$ if necessary, one may assume that $a<1$. We show that $f$ has a fixed point: pick an arbitrary point $x$ and consider the sequence $y_{k}=f^{k}(x)$. Then we have

$$
d\left(y_{i}, y_{i+1}\right)=d\left(f^{i}(x), f^{i+1}(x)\right)=a^{i} d(x, f(x)),
$$


which implies that the sequence is forward Cauchy. This sequence therefore has a unique limit $x_{0}$ and by continuity of $f$ we have

$$
f\left(x_{0}\right)=\lim _{j \rightarrow \infty} f\left(y_{j}\right)=\lim _{j \rightarrow \infty} y_{j+1}=x_{0},
$$

and thus found our fixed point $x_{0}$. We now consider the Binet-Legendre Riemannian metric $g_{F}$, by Theorem $1.2(\mathrm{~d})$, the mapping $f$ is a similarity also for $g_{F}$. We claim the following.

Lemma 6.2 Let $(M, g)$ be a $C^{0}$ Riemannian manifold. Assume that there exists a map $f: M \rightarrow M$ such that $d(f(x), f(y))=a \cdot d(x, y)$ for some constant $0<a<1$ where $d$ is the distance function corresponding to the Riemannian metric $g$. If $f$ has a fixed point, then $(M, g)$ is flat, ie, every point of $M$ has a neighborhood that is isometric to a domain in $\mathbb{R}^{n}$ with the standard metric.

As said before, we prove this lemma by a blow up argument ${ }^{1}$. Let $x_{0} \in M$ be the fixed point of $f$ and choose $R$ small enough so that the closed $d$-ball $\bar{B}_{R}\left(x_{0}\right)$ is compact. It suffices to show that the restriction of the metric $d$ to this ball is flat, since for every bounded neighborhood $U \subseteq M$ there exists $m$ such that $f^{m}(U) \subset B_{R}\left(x_{0}\right)$.

In order to do it, we construct a sequence of flat metrics $d_{m}$ on $B_{R}\left(x_{0}\right)$ such that it uniformly converges to the metric of $d$, in the sense that for every $x, y \in B_{R}\left(x_{0}\right)$ we have $d_{m}(x, y) \rightarrow d(x, y)$ uniformly as $m \rightarrow \infty$. Choosing a smaller radius $R$ if necessary, one may assume that some coordinates $x_{1}, \ldots, x_{n}$ are defined in some neighborhood of the ball $B_{R}\left(x_{0}\right)$. Assume also the point $x_{0}$ has coordinates $(0, \ldots, 0)$ and that the metric $g$ is given by the identity matrix at the point $x_{0}$. In this neighborhood, we consider the flat (constant) Riemannian metric $g_{0}=d x_{1}^{2}+\cdots+d x_{n}^{2}$. Both metrics $g$ and $g_{0}$ coincide at the point $x_{0}$. The distance in the metric $g$ is denoted by $d$ and that in the metric $g_{0}$ will be denoted by $d_{0}$. Likewise balls in the $d$-metric are denoted by $B_{r}(x)$ and balls in the $d_{0}$-metric will be denoted by $B_{r}^{\prime}(x)$.

We take $R^{\prime}$ such that $B_{R^{\prime}}^{\prime}\left(x_{0}\right) \subset B_{R}\left(x_{0}\right)$. For every $m \in \mathbb{N}$ we define a metric $d_{m}$ on $B_{R^{\prime}}^{\prime}\left(x_{0}\right)$ by

$$
d_{m}(x, y)=\frac{1}{a^{m}} d_{0}\left(f^{m}(x), f^{m}(y)\right) .
$$

Let us show that the sequence of metrics $d_{m}$ converges to the metric $d$. Since the metric $g$ is continuous, and since at the point $x_{0}$ the metric $g$ coincides with the metric $g_{0}$,

${ }^{1}$ The proof is elementary if the metric $g$ is $C^{2}$ : set $\kappa(x)=\max |K(\pi)|$ where $\pi$ ranges through all 2-planes in $T_{x} M$ and $K$ is the sectional curvature. For a similarity $f$ with dilation constant $a$ we have $\kappa(x)=a^{2 m} \kappa\left(f^{m}(x)\right)$ thus, if $a<1$ and $\left\{f^{m}(x)\right\}$ converges, we have $\kappa(x)=0$. 
for every $\varepsilon>0$ there exists $r(\varepsilon)$ such that for every point $x \in B_{3 r(\varepsilon)}^{\prime}\left(x_{0}\right) \cup B_{3 r(\varepsilon)}\left(x_{0}\right)$ and for every nonzero tangent vector $\xi \in T_{x} M$ we have

$$
\frac{1}{1+\varepsilon} \leq \frac{\sqrt{g(\xi, \xi)}}{\sqrt{g_{0}(\xi, \xi)}} \leq 1+\varepsilon .
$$

These inequalities immediately give the following estimates on the length of any curve $\gamma:[0,1] \rightarrow B_{3 r(\varepsilon)}\left(x_{0}\right):$

$$
\frac{1}{1+\varepsilon} L_{g}(\gamma) \leq L_{g_{0}}(\gamma) \leq(1+\varepsilon) L_{g}(\gamma)
$$

Assuming $\varepsilon<\frac{1}{2}$, these estimates imply that the shortest path connecting two points in $B_{r}\left(x_{0}\right)$ stays in the ball $B_{3 r}^{\prime}\left(x_{0}\right)$, and symmetrically the shortest path connecting two points in $B_{r}^{\prime}\left(x_{0}\right)$ stays in the ball $B_{3 r}\left(x_{0}\right)$. We therefore have the following inequalities for any $x, y \in B_{r(\varepsilon)}^{\prime}\left(x_{0}\right) \cap B_{r(\varepsilon)}\left(x_{0}\right)$ :

$$
\frac{1}{1+\varepsilon} d(x, y) \leq d_{0}(x, y) \leq(1+\varepsilon) d(x, y) .
$$

Now take two arbitrary points $x, y \in B_{R}\left(x_{0}\right)$. For sufficiently large $m$, the points $f^{m}(x)$ and $f^{m}(y)$ lie in $B_{r}(\varepsilon)\left(x_{0}\right)$. By definition, the distance between $f^{m}(x)$ and $f^{m}(y)$ is the length of a shortest curve. Since this curve lies in $B_{3 r(\varepsilon)}\left(x_{0}\right)$, the inequalities above imply that

$$
\frac{1}{1+\varepsilon} d\left(f^{m}(x), f^{m}(y)\right) \leq d_{0}\left(f^{m}(x), f^{m}(y)\right) \leq(1+\varepsilon) d\left(f^{m}(x), f^{m}(y)\right) .
$$

Dividing this inequality by $a^{m}$ and using the property $d\left(f^{m}(x), f^{m}(y)\right)=a^{m} \cdot d(x, y)$ together with the definition of $d_{m}$ we obtain

$$
\frac{1}{1+\varepsilon} d(x, y) \leq d_{m}(x, y) \leq(1+\varepsilon) d(x, y) .
$$

Since for $x, y \in B_{R}\left(x_{0}\right)$ the function $d(x, y)$ is uniformly bounded by $2 R$, the metrics $d_{m}$ uniformly converge to the metric $d$ as $m \rightarrow \infty$. Furthermore the metrics $d_{m}$ are clearly flat metrics: $B_{R}\left(x_{0}\right)$ equipped with such metric is isometric to a domain in the standard Euclidean space $\mathbb{R}^{n}$.

Is it is well known that a uniform limit of flat metrics, is itself flat. For the sake of completeness, we give a proof of this fact in our case. We may assume that $R \geq 3$, otherwise we divide the metric by a large constant. We will prove that the metric $d$ in the ball $B_{1}\left(x_{0}\right)$ is flat. 
For any $m$, we choose an isometric embedding $\phi_{m}:\left(\bar{B}_{R}\left(x_{0}\right), d_{m}\right) \rightarrow \mathbb{R}^{n}$ such that $\phi_{m}\left(x_{0}\right)=0$. Let us set $x_{j}(m)=\phi_{m}^{-1}\left(e_{i}\right) \in \bar{B}_{R}\left(x_{0}\right)$ where $e_{1}, e_{2}, \ldots, e_{n} \in \mathbb{R}^{n}$ is the standard orthonormal basis.

Since $\bar{B}_{R}\left(x_{0}\right)$ is compact, one can find a subsequence $\left(x_{1}\left(m_{i}\right), \ldots, x_{n}\left(m_{i}\right)\right)$ converging to a tuple $\left(x_{1}, \ldots, x_{n}\right) \in B_{1}\left(x_{0}\right) \times \cdots \times B_{1}\left(x_{0}\right)$. We claim that the restriction of the sequence $\phi_{m_{i}}$ to $B_{1}\left(x_{0}\right)$ converges to a map $\phi: B_{1}\left(x_{0}\right) \rightarrow \mathbb{R}^{n}$ which is an isometry. Indeed, for any $y \in \bar{B}_{R}\left(x_{0}\right)$ the point $\phi_{m_{i}}(y)$ is the unique point in $\mathbb{R}^{n}$ such that $\left\|\phi_{m_{i}}(y)\right\|=d_{m_{i}}\left(x_{0}, y\right)$ and $\left\|\phi_{m_{i}}(y)-e_{j}\right\|=d_{m}\left(x_{j}, y\right)$ for any $j=1, \ldots, n$. Since the sequence $x_{j}\left(m_{i}\right)$ converges to $x_{i}$ and $d_{m_{i}}$ converges uniformly to $d$, the sequence $\left\{\phi_{m_{i}}(y)\right\}$ converges to the unique point $Y \in \mathbb{R}^{n}$ such that $\|Y\|=d\left(x_{0}, y\right)$ and $\left\|Y-e_{j}\right\|=d\left(x_{j}, y\right)$ for any $j=1, \ldots, n$.

We denote by $\phi=\lim _{i \rightarrow \infty} \phi_{m_{i}}$ the limiting map. This is an isometry since

$$
d\left(y, y^{\prime}\right)=\lim _{i \rightarrow \infty} d_{m_{i}}(y, y)=\lim _{i \rightarrow \infty}\left\|\phi_{m_{i}}(y)-\phi_{m_{i}}\left(y^{\prime}\right)\right\|=\left\|\phi(y)-\phi\left(y^{\prime}\right)\right\| .
$$

The proof of Lemma 6.2 is complete.

The lemma just proved tells us that a neighborhood of the point $x_{0} \in M$ equipped with the metric $g_{F}$ is isometric to a domain in the standard Euclidean space. The next lemma (which provides the second step in the proof of Theorem 6.1) says that the metric $F$ is isometric to a Minkowski metric in the same neighborhood.

Lemma 6.3 Let $F$ be a Finsler metric on a domain $U \subseteq \mathbb{R}^{n}$ and let $f: U \longrightarrow U$ be a map which is a self-similarity with dilation constant $a<1$ for both the Finsler metric $F$ and the standard Euclidean metric $g$ on $\mathbb{R}^{n}$. If $f$ has a fixed point, then $F$ is (the restriction of) a Minkowski metric.

Note in the lemma we neither suppose $F$ is complete nor that it is quasireversible.

To prove this lemma, assume that $U$ contains the origin and that 0 is the fixed point. Then $f$ is the restriction of a linear similarity (still denoted by $f: \mathbb{R}^{n} \rightarrow \mathbb{R}^{n}$ ) and has thus the form $f(x)=a \cdot Q(x)$, for some orthogonal transformation $Q \in O(n)$. By hypothesis, we have

$$
F\left(f(x), d f_{x}(\xi)\right)=f^{*} F(x, \xi)=a \cdot F(x, \xi)
$$

for any $(x, \xi) \in T \mathbb{R}^{n}=\mathbb{R}^{n} \times \mathbb{R}^{n}$. Because $\left.d f_{x}(\xi)\right)=a \cdot Q(\xi)$, we have

$$
F\left(f(x), d f_{x}(\xi)\right)=F(f(x), a \cdot Q(\xi))=a \cdot F(f(x), Q(\xi)) .
$$

It follows from the two previous equalities that

$$
F(x, \xi)=F(f(x), Q(\xi))=a^{n} F\left(f^{n}(x), Q^{n}(\xi)\right)
$$


for any integer $n$. Fix an arbitrary point $x \in \mathbb{R}^{n}$ and choose a sequence $\left\{n_{j}\right\} \subset \mathbb{N}$ such that $Q^{n_{j}} \longrightarrow$ id in $O(n)$ as $j \rightarrow \infty$, we then have

$$
F(x, \xi)=\lim _{k \rightarrow \infty} F\left(f^{n_{k}}(x), Q^{n_{k}}(\xi)\right)=F(0, \xi) .
$$

This shows that $F(x, \xi)$ is independent of $x$, ie, it is a Minkowski metric. The second lemma is proved.

We can now conclude the proof of Theorem 6.1. By Lemmas 6.2 and 6.3 the metric $F$ is a Minkowski metric in a certain neighborhood $U$ of $x_{0}$. Since for bounded set $U^{\prime} \subset M$ there exists $m$ such that $f^{m}\left(U^{\prime}\right) \subset U$, the metric $F$ is a Minkowski metric in some neighborhood of every point. Clearly, $M$ is simply connected. Indeed, for every loop $\gamma$ there exists $m$ such that $f^{m}(\gamma)$ lies in a small neighborhood of $x_{0}$ and is therefore contractible. Because $f^{m}$ is a homeomorphism on its image, the loop $\gamma$ is contractible as well. We established that the manifold $(M, F)$ is forward complete, simply connected and locally isometric to a Minkowski space; it is therefore globally isometric to a Minkowski space.

In the case of smooth Finsler manifolds, Theorem 6.1 is known. A first proof was given by Heil and Laugwitz in [19], however R L Lovas and J Szilasi found a gap in the argument and gave a new proof in [29].

\section{Conformal transformations of (partially smooth) Finsler metrics}

In this section, we classify all conformal transformations of an arbitrary Finsler manifold.

Definition 7.1 A set $S \subseteq \operatorname{Diff}(M)$ of transformations of the Finsler manifold $(M, F)$ is said to be essentially conformal if any $f \in S$ is a conformal transformation of $(M, F)$, but there is no conformal deformation $\lambda \cdot F$ of $F$ for which $S$ is a set of isometries. The set $S$ of conformal transformations of $M$ is termed inessential if it is not essentially conformal.

Theorem 7.2 Let $(M, F)$ be a connected $C^{\infty}$ partially smooth Finsler manifold. Then the following conditions are equivalent:

(a) There exists an essentially conformal diffeomorphism $f$ of $(M, F)$.

(b) The group of conformal diffeomorphisms of $(M, F)$ is essential.

(c) $(M, F)$ is conformally equivalent to a Minkowski space $\left(\mathbb{R}^{n}, F\right)$ or to the canonical Riemannian sphere $\left(\mathbb{S}^{n}, g_{0}\right)$. 
The logic of the proof is the following: Using the Binet-Legendre construction, we reduce this theorem to the Alekseevskiu-Ferrand-Schoen solution to the Riemannian Lichnerowicz-Obata conjecture; see eg Alekseevskiü [1], Ferrand [15] and Schoen [40]. We then need to prove that the Finsler metric is conformally Minkowski in the noncompact case and Riemannian in the compact case. The main ideas are similar to those in [35], but here we do not work with conformal vector fields.

Note it is obvious that (a) $\Rightarrow$ (b), but (b) $\Rightarrow$ (a) is not a priori a trivial fact because we could conceive of a Finsler manifold $(M, F)$ for which every conformal diffeomorphism would be inessential, but for which no conformal deformation $\lambda \cdot F$ of the metric would be simultaneously invariant under all conformal diffeomorphisms of $(M, F)$.

Proof As just observed, (a) trivially implies (b). It is also clear that (c) $\Rightarrow$ (a), since any linear contraction of a Minkowski space and any nonisometric Möbius transformation of the sphere are examples of essential conformal transformations. We thus only need to prove (b) $\Rightarrow$ (c).

We know from Theorem 1.2(d) that $f$ is also a conformal transformation for the associated Binet-Legendre metric, and $f:\left(M, g_{F}\right) \rightarrow\left(M, g_{F}\right)$ must be essential otherwise $f$ would be an inessential conformal transformation of $(M, F)$.

It follows that the full group of conformal transformations of $\left(M, g_{F}\right)$ is essential and by the Alekseevskiu-Ferrand-Schoen Theorem, the manifold $\left(M, g_{F}\right)$ is either conformally equivalent to the Euclidean space $\mathbb{R}^{n}$ or to the canonical Riemannian sphere $\mathbb{S}^{n}$. Changing the Finsler metric $F$ and correspondingly the Binet-Legendre metric $g_{F}$ within the same conformal class, we will assume that $\left(M, g_{F}\right)$ is in fact isometric to $\mathbb{R}^{n}$ or $\mathbb{S}^{n}$.

If $\left(M, g_{F}\right)$ is isometric to the Euclidean space $\left(\mathbb{R}^{n}, g_{0}\right)$, then $f$ is a conformal transformation of $\mathbb{R}^{n}$ and it is therefore a map of the type $f(x)=a \cdot Q(X)+b$ with $Q \in O(n), a>0$ and $b \in \mathbb{R}^{n}$. Since $f$ is essential, we have $a \neq 1$ and we conclude from Lemma 6.3 that $F$ is a Minkowski metric. Our claim is proved in this case.

We now assume that $\left(M, g_{F}\right)$ is isometric to the canonical Riemannian sphere $\mathbb{S}^{n}$ and $f: \mathbb{S}^{n} \rightarrow \mathbb{S}^{n}$ is a nonisometric conformal map. It is well known that such a map has exactly either one or two fixed points.

Case 1 ( $f$ has two fixed points) Using a stereographic projection, we identify $\mathbb{S}^{n}$ with $\mathbb{R}^{n} \cup\{\infty\}$ and we may assume that $f(\infty)=\infty$ and $f(0)=0$. Thus $f$ induces a conformal map $f: \mathbb{R}^{n} \rightarrow \mathbb{R}^{n}$ which is of the type $f(x)=a \cdot Q(x)$, with $Q \in O(n)$. If $a=1$, then $f$ is an isometry of the spherical metric

$$
g_{1}=\rho^{2}(x) \cdot g_{0}, \quad \rho(x)=\frac{2}{1+|x|^{2}},
$$


where $g_{0}=\sum d x_{i}^{2}$ is the standard Euclidean metric. By hypothesis this metric $g_{1}$ coincides with the Binet-Legendre metric $g_{F}$ of $F$, and by Theorem 1.2(d), the map $f$ is then also an isometry of the Finsler metric $F$, ie $f$ is inessential, a case that we excluded.

So we have $a \neq 1$. Consider the Finsler metric $F^{+}=\rho^{-1} \cdot F$ on $\mathbb{R}^{n}$, its Binet-Legendre metric is the flat metric $g_{0}=\rho^{-2} \cdot g_{1}$. The map $f(x)=a \cdot Q(x)$ is a nonisometric similarity for both the Binet-Legendre metric $g_{F^{+}}=g_{0}$ and the Finsler metric $F^{+}$ and we conclude from Lemma 6.3 that $F^{+}$is a Minkowski metric.

Let $\varphi(x)=x /|x|^{2}$ be the standard inversion in $\mathbb{R}^{n} \cup\{\infty\}$. This map exchanges the two fixed points of $f$ and the previous argument shows that $F^{-}=\rho^{-1} \cdot \varphi^{*} F$ is also a Minkowski metric. Since $\varphi$ is conformal for the Binet-Legendre metrics of $F^{+}$and $F^{-}$, the Liouville Theorem (Theorem 4.1) implies that $F^{+}=\rho^{-1} \cdot F$ is a Euclidean metric $g^{+}$and thus $F$ is Riemannian. Hence $F(\xi)=\sqrt{g_{F}(\xi, \xi)}$ is the standard metric on $\mathbb{S}^{n}$.

Case 2 ( $f$ has exactly one fixed point) We again identify $\mathbb{S}^{n}$ with $\mathbb{R}^{n} \cup\{\infty\}$ and assume that $f(\infty)=\infty$. Thus $f$ induces a conformal map $f: \mathbb{R}^{n} \rightarrow \mathbb{R}^{n}$ which is of the type $f(x)=a \cdot Q(X)+b$. Since $f$ has no fixed point in $\mathbb{R}^{n}$, we must have $b \neq 0$ and $a=1$. Using Lemma 7.3 below and conjugating $f$ with a translation if necessary, we may assume that $b$ is an eigenvector of $Q$ with eigenvalue +1 , ie $Q(b)=b$.

We are thus in the following situation: our map $f$ is $f(x)=Q(x)+b$ where $Q(b)=b \neq 0$ and the composition $\tilde{f}=\varphi \circ f: \mathbb{R}^{n} \backslash\{0\} \rightarrow \mathbb{R}^{n}$ is conformal for the standard metric, where $\varphi$ is the inversion. We have

$$
\begin{aligned}
\tilde{f}(x)=\frac{Q(x)+b}{|Q(x)+b|^{2}}, \\
d \tilde{f}_{x}(\xi)=\frac{Q(\xi)}{|Q(x)+b|^{2}}-2\langle Q(\xi), Q(x)+b\rangle \frac{Q(x)+b}{|Q(x)+b|^{4}} \\
=\frac{1}{|f(x)|^{2}}\left(Q(\xi)-2 \frac{\langle Q(\xi), f(x)\rangle}{|f(x)|^{2}} \cdot f(x)\right) \\
=\frac{1}{|f(x)|^{2}} \cdot\left(S_{f(x)} \circ Q\right)(\xi),
\end{aligned}
$$

where $S_{f(x)}$ is the linear reflection across the hyperplane $f(x)^{\perp}$. Since $Q(b)=b$, we have $f^{n}(x)=Q^{n}(x)+n \cdot b$, and the same calculation gives us

$$
d\left(\tilde{f}_{n}\right)_{x}(\xi)=\frac{1}{\left|f^{n}(x)\right|^{2}} \cdot\left(S_{f(x)^{n}} \circ Q^{n}\right)(\xi),
$$


for any $n \in \mathbb{N}$, where $\tilde{f}_{n}=\varphi \circ f^{n}$. The map $\tilde{f}_{n}$ is conformal for the Finsler metric $F$, we thus have

$$
F\left(\tilde{f}_{n}(x), d\left(\tilde{f}_{n}\right)_{x}(\xi)\right)=\lambda_{n}(x) \cdot F(x, \xi)
$$

for some function $\lambda_{n}$, therefore

$$
\begin{aligned}
F(x, \xi) & =\frac{1}{\lambda_{n}(x)} \cdot F\left(\tilde{f}_{n}(x), d\left(\tilde{f}_{n}\right)_{x}(\xi)\right) \\
& =\mu_{n}(x) \cdot F\left(\tilde{f}_{n}(x),\left(S_{f(x)^{n}} \circ Q^{n}\right)(\xi)\right),
\end{aligned}
$$

where $\mu_{n}(x)=1 /\left(\left|f^{n}(x)\right|^{2} \lambda_{n}(x)\right)$. Observe that $S_{f(x)^{n}}$ only depends on the direction of the vector $f^{n}(x)$, ie $S_{f(x)^{n}}=S_{\frac{f(x)^{n}}{\left|f(x)^{n}\right|}}$, and since

$$
\lim _{n \rightarrow \infty} \frac{f(x)^{n}}{\left|f(x)^{n}\right|}=\lim _{n \rightarrow \infty} \frac{Q^{n}(x)+n \cdot b}{\left|Q^{n}(x)+n \cdot b\right|}=\frac{b}{|b|},
$$

we have

$$
\lim _{n \rightarrow \infty} S_{f(x)^{n}}=S_{b}
$$

By the compactness of the group $O(n)$, one may find a sequence $\left\{n_{j}\right\} \subset \mathbb{N}$ such that $Q^{n_{j}} \rightarrow I$, we thus have

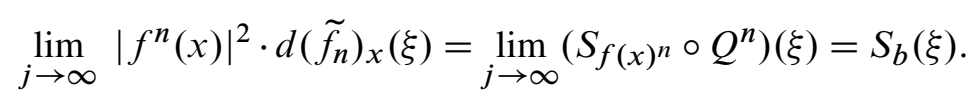

Now $\mu_{n}(x)$ is a bounded sequence and we may choose the subsequence $\left\{n_{j}\right\}$ such that $\mu_{n_{j}}(x)$ converges to some number $\mu(x)$. The previous considerations imply that

$$
F(x, \xi)=\lim _{j \rightarrow \infty} \mu_{n_{j}}(x) \cdot F\left(\tilde{f}_{n_{j}}(x),\left(S_{f(x)^{n} \circ Q^{n_{j}}}\right)(\xi)\right)=\mu(x) \cdot F\left(0, S_{b}(\xi)\right)
$$

for any $(x, \xi)$. It follows that $\frac{1}{\mu} F$ is a Minkowski metric.

Since the inversion $\varphi$ is conformal for the Minkowski metric $\frac{1}{\mu} F$, the Liouville Theorem (Theorem 4.1) implies $F$ is in fact a Riemannian metric and thus $F(\xi)=\sqrt{g_{F}(\xi, \xi)}$ is the standard metric on $\mathbb{S}^{n}$.

Lemma 7.3 Suppose that $f(x)=Q(x)+b$ is a fixed point free transformation of $\mathbb{R}^{n}$ with $Q \in O(n)$. Then $f$ can be decomposed as

$$
f=T \circ f_{1} \circ T^{-1},
$$

where $T$ is a translation and $f_{1}(x)=Q(x)+b_{1}$ for some nonzero vector $b_{1}$ such that $Q\left(b_{1}\right)=b_{1}$. 
Proof Let us denote by $E=\left\{v \in \mathbb{R}^{n} \mid Q(v)=v\right\}$. The decomposition $\mathbb{R}^{n}=E \oplus E^{\perp}$ is $Q$-invariant and we write $b=b_{1}+b_{2}$ with $b_{1} \in E$ and $b_{2} \in E^{\perp}$. The transformation $f_{2}(x)=Q(x)+b_{2}$ has a fixed point $v_{0} \in E^{\perp}$. Indeed, 1 is not an eigenvalue of the restriction $\left.Q\right|_{E \perp}$, therefore the equation

$$
(Q-I)(v)=-b_{2}, \quad v \in E^{\perp}
$$

has a solution $v_{0}$, and we have $Q\left(v_{0}\right)+b_{2}=v_{0}$. Let us denote by $T$ the translation $T(x)=x+v_{0}$. We then have

$$
\begin{aligned}
\left(T^{-1} \circ f \circ T\right)(x) & =\left(Q\left(x+v_{0}\right)+b\right)-v_{0} \\
& =Q(x)+(Q-I)\left(v_{0}\right)+\left(b_{1}+b_{2}\right) \\
& =Q(x)+b_{1} .
\end{aligned}
$$

It is clear that $Q\left(b_{1}\right)=b_{1}$ since $b_{1} \in E$, and $b_{1} \neq 0$, otherwise $f(x)=Q(x)+b_{2}$ would have a fixed point.

\section{On Berwald spaces}

A $C^{k}$-Berwald space is a Finsler manifold $(M, F)$ which admits a torsion free linear connection $\nabla$ which is compatible with the Finsler metric. More precisely, one says that a linear connection $\nabla$ on a smooth manifold is of class $C^{k}$ if its Christoffel symbols in any coordinate system are of class $C^{k}$. Recall that the parallel transport associated to a $C^{1}$-path $\gamma:[0,1] \rightarrow M$ from $x=\gamma(0)$ to $y=\gamma(1)$ is the linear map $P_{\gamma}: T_{x} M \rightarrow T_{y} M$ defined as $P_{\gamma}\left(\xi_{t}\right)=\xi_{1} \in T_{y} M$ where $t \rightarrow \xi_{t}$ is the solution to the equation $\nabla_{\dot{\gamma}(t)} \xi_{t}=0$ such that $\xi_{0}=\xi \in T_{x} M$. Observe that, since this ordinary differential equation is linear in $\xi_{t}$, there is a unique solution for any $t \in[0,1]$ even when the connection $\nabla$ is only of class $C^{0}$ (see Hartman [18]).

Definition 8.1 A Finsler metric $F$ on a manifold $M$ is said to be a $C^{k}$-Berwald metric if there exists a $C^{k}$-smooth torsion free linear connection $\nabla$ (called an associated connection) on $M$ whose associated parallel transport preserves the Lagrangian $F$. That is, if $\gamma:[0,1] \rightarrow M$ is a smooth path connecting the point $x=\gamma(0)$ to $y=\gamma(1)$ and $P_{\gamma}: T_{x} M \rightarrow T_{y} M$ is the associated $\nabla$-parallel transport, then

$$
F\left(y, P_{\gamma}(\xi)\right)=F(x, \xi)
$$

for any $\xi \in T_{x} M$.

Observe that if an associated connection $\nabla$ of a Berwald metric $F$ is of class $C^{k}$, then the metric $F$ is $C^{k}$-partially smooth. 
Note that the definition given here differs (and is more general) from that given by Bao, Chern and Shen in [3], but both definitions are equivalent for $C^{2}$ and strongly convex Finsler metrics; see Chern and Shen [10, Proposition 4.3.3].

In 1981, Z I Szabò proved that for a smooth and strongly convex Berwald metric, there exists an associated connection which is the Levi-Civita connection of some Riemannian metric on $M$. Later, other proofs that do not require strict convexity were given by the first author in [34] and by Vincze in [46]. Our next result, whose proof is very simple, extends Szabò's theorem to the case of $C^{0}$ Finsler metrics.

Theorem 8.2 Let $(M, F)$ be a $C^{0}$-Berwald-Finsler manifold. If $\nabla$ is an associated connection, then the parallel transport associated to the connection $\nabla$ preserves the Binet-Legendre metric $g_{F}$.

Proof For any smooth path $\gamma:[0,1] \rightarrow M$, the parallel transport $P_{\gamma}: T_{x} M \rightarrow T_{y} M$ is a linear map that sends the unit ball of $F$ at $x=\gamma(0)$ to the unit ball of $F$ at $y=\gamma(1)$. By Proposition 12.1(b), the parallel transport preserves the Binet-Legendre metric $g_{F}$ as we claim.

Remark (A) The theorem implies the following extension of Szabò's theorem: Any partially $C^{1}$-Berwald metric has a unique associated linear connection $\nabla$ and this connection is the Levi-Civita connection of the Binet-Legendre metric $g_{F}$.

(B) One may in fact redefine a partially smooth Berwald metric as a Finsler metric for which the Levi-Civita connection of the Binet-Legendre metric preserves $F$.

(C) Observe that a Finsler manifold $(M, F)$ is flat (ie locally Minkowski) if and only if it is Berwald and $g_{F}$ is a flat Riemannian metric.

(D) It is now easy to produce examples of non-Berwald metrics for which all tangent spaces $T_{x} M$ are isometric as Minkowski spaces (such Finsler metrics are called monochromatic by Bao in [2, Section 3.3]). Take a non-Euclidean Minkowski metric $F_{0}$ on $\mathbb{R}^{n}$ and let $A$ be a smooth field of endomorphisms such that for every point $x$ the endomorphism $A_{x}$ is an orthogonal transformation for the Binet-Legendre metric: $A_{x} \in O\left(\mathbb{R}^{n}, g_{F}\right)$. Let $\widetilde{F}(x, \xi)=F_{0}\left(A_{x}(\xi)\right)$, by construction $F$ and $\widetilde{F}$ have the same Binet-Legendre metric. In particular $g_{\widetilde{F}}$ is flat, and all tangent spaces are isometric to $F_{0}$, but unless $A_{x}$ is an isometry of $F_{x}$ (for any point $x$ ), the Finsler metric $\widetilde{F}$ is not Berwald.

(E) One can describe all partially smooth Berwald spaces by the following construction. Choose an arbitrary smooth Riemannian metric $g$ on $M$ and choose an arbitrary Minkowski norm in the tangent space at some fixed point $q$ that is invariant with respect to the holonomy group of $g$. Now extend this norm to 
all other tangent spaces by parallel translation with respect to the Levi-Civita connection of $g$. Since the norm is invariant with respect to the holonomy group, the extension does not depend on the choice of the curve connecting an arbitrary point to $q$, and is a partially smooth Berwald-Finsler metric.

We see that if the holonomy group of $g_{F}$ acts transitively on the unit sphere in some tangent space, then the Finsler metric $F$ is actually Riemannian. When the holonomy group is not transitive, we have the following result.

Proposition 8.3 Let $F$ be a $C^{2}$-partially smooth non-Riemannian Berwald metric on a connected manifold $M$. Then, either there exists another Riemannian metric $h$ which is affinely equivalent to $g_{F}$ but not proportional to $g_{F}$, or the metric $\left(M, g_{F}\right)$ is symmetric of rank greater than or equal to 2 , or both.

Recall that a Riemannian symmetric space $(M, g)$ is said to be of rank $k$ if every point belongs to a subspace $E^{k} \subset M$ which is isometric to the Euclidean space $\mathbb{R}^{k}$.

Remark Recall that by de Rham's Splitting Theorem [11], the existence of $h$ such that it is not proportional to $g_{F}$, but is affine equivalent to $g_{F}$, implies that $\left(M, g_{F}\right)$ is locally decomposable, in the sense that every point of it has a neighborhood $U$ that is isometric to the direct product of two Riemannian manifolds of positive dimensions. If in addition $\left(M, g_{F}\right)$ is complete, the universal cover of $\left(M, g_{F}\right)$ is the direct product of two complete Riemannian manifolds of positive dimensions.

Proof We essentially repeat the argumentation in $[34 ; 46 ; 43]$. Fix a point $q \in M$. For every smooth loop $\gamma(t),(0 \leq t \leq 1)$ such that $\gamma(0)=\gamma(1)=q$, we denote by $P_{\gamma}: T_{q} M \rightarrow T_{q} M$ the parallel transport along that loop with respect to the Levi-Civita connection of $g$. The set

$$
H_{q}=\left\{P_{\gamma} \mid \gamma:[0,1] \rightarrow M \text { smooth, } \gamma(0)=\gamma(1)=q\right\}
$$

is a subgroup of the group of the orthogonal transformations of $\left(T_{q} M, g_{F}\right)$. Moreover, it is well known (see for example, Berger [5] and Simons [42]), that at least one of the following conditions holds:

(1) $H_{q}$ acts transitively on $S_{1}=\left\{\xi \in T_{q} M \mid g(\xi, \xi)=1\right\}$.

(2) The metric $g_{F}$ is symmetric of rank $\geq 2$.

(3) There exists another Riemannian metric $h$ which is affinely equivalent but not proportional to $g_{F}$.

In the first case, the ratio $F(\xi) / \sqrt{g_{F}(\xi, \xi)}$ is a constant function on the sphere $T_{q} M \backslash\{0\}$, implying the metric $F$ is Riemannian, which is contrary to our hypothesis. Thus either the second or the third case holds and the proposition is proved. 


\section{On locally symmetric Finsler spaces}

Definition 9.1 The Finsler manifold $(M, F)$ is called locally symmetric, if for every point $x \in M$ there exists $r=r(x)>0$ and an isometry $\tilde{I}_{x}: B_{r}(x) \rightarrow B_{r}(x)$ (called the reflection at $x)$ such that $\tilde{I}_{x}(x)=x$ and $d_{x}\left(\tilde{I}_{x}\right)=-\mathrm{id}: T_{x} M \rightarrow T_{x} M$. The largest $r(x)$ satisfying this condition is called the symmetry radius at $x$. The manifold $(M, F)$ is called globally symmetric if the reflection $\tilde{I}_{x}$ can be extended to a global isometry: $\tilde{I}_{x}: M \rightarrow M$.

Theorem 9.2 Let $(M, F)$ be a $C^{2}$-smooth Finsler manifold. If $(M, F)$ is locally symmetric, then $F$ is $C^{\infty}$-Berwald ${ }^{2}$.

Remark 9.3 This theorem answers positively a conjecture stated in [14], where it has been proved for globally symmetric spaces; see also Busemann [6, Section 49], Foulon [16] and Kim [22].

Proof We will first prove the theorem under the additional assumption that the metric $F$ is strongly convex. Since every local isometry for the Finsler metric $F$ is also an isometry for the Binet-Legendre metric $g_{F}$, it follows that $\left(M, g_{F}\right)$ is a Riemannian locally symmetric space.

In what follows, it will be convenient to use tilde-notation for the "Finsler" objects, and the nontilde notation for the analogous objects for the Binet-Legendre metric $g_{F}$ (for example $B_{r}(x)$ will denote the $r$-ball in $g_{F}$, and $\widetilde{B}_{r}(x)$ the $r$-ball in $F ; \gamma(t)$ will denote $g_{F}$-geodesic and $\tilde{\gamma}(t)$ will denote $F$-geodesics). Note that a locally symmetric space is evidently reversible, so that the distance function in $F$ is symmetric, and if $t \mapsto \tilde{\gamma}(t)$ is a geodesic parameterized by arc length, the reversed curve $t \mapsto \tilde{\gamma}(-t)$ is also a geodesic parameterized by arc length.

It is known that a locally symmetric Riemannian manifold is locally isometric to a globally symmetric space (see Helgason [20, theorem 5.1]) and is therefore real analytic. Then, for sufficiently small neighborhood $W \subset M$ and for every $x \in W$, the $g_{F}$-reflection $I_{x}$ is defined globally on $W$.

For every $x \in W$, there is also the reflection $\widetilde{I}_{x}: \widetilde{B}_{\widetilde{r}(x)}(x) \rightarrow \widetilde{B}_{\widetilde{r}(x)}(x)$ for the Finsler metric. By Theorem 1.2(d), the Finsler reflection $\widetilde{I}_{x}$ coincides with the restriction of the Riemannian reflection $I_{x}$ on $\widetilde{B}_{\widetilde{r}(x)}(x) \cap W$. We do not know ${ }^{3}$ a priori whether $I_{x}$ is an $F$-isometry in the whole ball $B_{\rho}(x)$.

\footnotetext{
${ }^{2}$ According to Definition 8.1 , it means that the associated connection $\nabla$ is $C^{\infty}$-smooth. We can in fact prove that the associated connection is $C^{\omega}$; but this does not imply that the metric $F$ itself is $C^{\infty}$.

${ }^{3}$ A different definition of locally symmetric Finsler manifolds was given by Berestovskiŭ in [4] and Busemann and Phadke in [7]: it was explicitly assumed that the radius of symmetry $\widetilde{r}(x)$ was locally
} 
Claim For every sufficiently small $g_{F}$-geodesically convex open set $W \subset M$ and for every $F$-geodesic $\tilde{\gamma}(t):[-\widetilde{\varepsilon}, \widetilde{\varepsilon}] \rightarrow W$ parameterized by arc length, we have $I_{\widetilde{\gamma}(0)}(\widetilde{\gamma}(t))=\tilde{\gamma}(-t)$ for all $t \in[-\widetilde{\varepsilon}, \widetilde{\varepsilon}]$.

Recall that $W$ is $g_{F}$-geodesically convex, if every pair of points in $W$ can be connected by a unique minimal $g_{F}$-geodesic and that geodesic lies in $W$. To prove the claim, we take a $F$-geodesic $\tilde{\gamma}:[-\widetilde{\varepsilon}, \widetilde{\varepsilon}] \rightarrow W$, set $x=\tilde{\gamma}(0) \in W$, consider the $g_{F}$-reflection $I_{x}$ and the number

$$
r_{0}(\tilde{\gamma}, x)=\sup \left\{r^{\prime} \in[0, \widetilde{\varepsilon}] \mid I_{x}(\tilde{\gamma}(t))=\tilde{\gamma}(-t) \text { for all } t \in\left[-r^{\prime}, r^{\prime}\right]\right\} .
$$

Since the metric $F$ is strongly convex, there is a unique $F$-geodesic with any given initial vector. Then, because $I_{x} \equiv \tilde{I}_{x}$ in a small neighborhood of $x$, we have $r_{0}(\tilde{\gamma}, x)>0$. We want to prove that $r_{0}(\tilde{\gamma}, x)=\widetilde{\varepsilon}$. Let us assume that $r_{0}(\tilde{\gamma}, x)<\widetilde{\varepsilon}$ and derive a contradiction.

Indeed, set $x_{+}=\tilde{\gamma}\left(r_{0}\right)$ and $x_{-}=\tilde{\gamma}\left(-r_{0}\right)$ and consider (the analytical continuation of) the $g_{F}$-reflections $I_{x_{+}}, I_{x}, I_{x_{-}}$. Consider $I_{x_{-}} \circ I_{x} \circ I_{x_{+}}$. It is again a $g_{F}$ isometry. Let us show that it coincides with $I_{x}$. In order to do this, we consider the $g_{F}$-geodesic $\gamma(t)$ containing $x_{+}=\tilde{\gamma}\left(r_{0}\right)$ and $x_{-}=\tilde{\gamma}\left(-r_{0}\right)$. Reparameterizing this geodesic affinely if necessary, we may assume without loss of generality that $\gamma(1)=x_{+}$ and $\gamma(-1)=x_{-}$. Since the neighborhood $W$ is sufficiently small, we may assume $\gamma$ is defined at least on $[-2,2]$. Since $I_{x}\left(x_{+}\right)=x_{-} \in W$ and $I_{x}\left(x_{-}\right)=x_{+} \in W$, we have that $I_{x}(\gamma)$ is a shortest $g_{F}$-geodesic connecting $x_{+}$to $x_{-}$. By convexity of $W$, we must have $I_{x}(\gamma) \subset W$ and $I_{x}(\gamma(t))=\gamma(-t)$. In particular $I_{x}(\gamma(0))=\gamma(0)$. By uniqueness of the fixed point of $I_{x}$ in a geodesically convex region, it follows that $\gamma(0)=x$. Now, we have

$$
I_{x_{+}}(x)=\gamma(2), \quad I_{x}(\gamma(2))=\gamma(-2), \quad I_{x_{-}}(\gamma(-2))=\gamma(0)=x .
$$

This implies $I_{x_{-}} \circ I_{x} \circ I_{x_{+}}(x)=x=I_{x}(x)$. We next show $d_{x}\left(I_{x_{-}} \circ I_{x} \circ I_{x_{+}}\right)=-\mathrm{id}$. Choose a vector $\xi \in T_{x} M$ and extend it as parallel vector field along the geodesic $\gamma$. Since the reflection $I_{x_{-}}$leaves $\gamma$ invariant and satisfies $d_{x_{-}}\left(\xi_{x_{-}}\right)=-\xi_{x_{-}}$, and since an isometry preserves parallel vector fields, we have $\left(I_{x_{-}}\right)_{*}(\xi)=-\xi$ at every point of $\gamma$. The same holds for the reflections $I_{x}$ and $I_{x_{+}}$, therefore $\left(I_{x_{-}} \circ I_{x} \circ I_{x_{+}}\right)_{*} \xi=-\xi$ (for arbitrary $\left.\xi \in T_{x} M\right)$. It follows that $d_{x}\left(I_{x_{-}} \circ I_{x} \circ I_{x_{+}}\right)=-\mathrm{id}=d_{x} I_{x}$ and therefore $I_{x_{-}} \circ I_{x} \circ I_{x_{+}}=I_{x}$.

Now, for $\underset{\widetilde{B}}{\delta}>0$ small enough, the mappings $I_{x_{-}}$and $I_{x_{+}}$are $F$-isometries in the $F$-balls $\widetilde{B}_{\delta}\left(x_{-}\right)$and $\widetilde{B}_{\delta}\left(x_{+}\right)$, respectively; see Figure 2.

bounded below. Under this assumption, $I_{x}$ coincides with $\widetilde{I}_{x}$ in the whole $\widetilde{B}_{r}$, where $r$ can be universally 


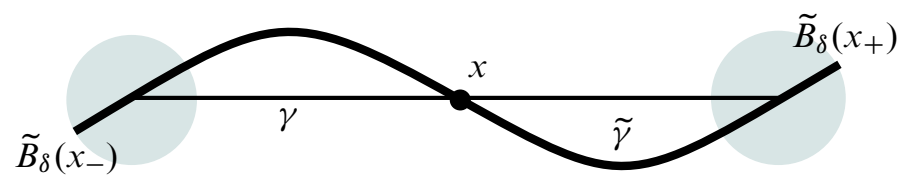

Figure 2: The geodesics $\gamma, \tilde{\gamma}$ and the balls $\widetilde{B}_{\delta}\left(x_{-}\right), \widetilde{B}_{\delta}\left(x_{+}\right)$.

Using again the uniqueness of an $F$-geodesic with prescribed given initial vector, we see that the mapping $I_{x_{-}} \circ I_{X} \circ I_{X_{+}}$sends the $F$-geodesic segment $\tilde{\gamma}_{\mid\left[r_{0}, r_{0}+\delta\right]}$ to the $F$-geodesic segment $\tilde{\gamma}_{\mid\left[-r_{0}-\delta,-r_{0}\right]}$. Replacing the isometry $I_{x_{-}} \circ I_{x} \circ I_{x_{+}}$by the isometry $I_{x_{+}} \circ I_{x} \circ I_{x_{-}}$in the previous argument, we obtain that $I_{x_{+}} \circ I_{x} \circ I_{x_{-}}$sends

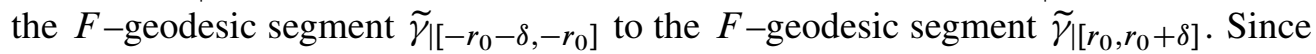
$I_{x_{-}} \circ I_{x} \circ I_{x_{+}}=I_{x}=I_{x_{+}} \circ I_{x} \circ I_{x_{-}}$, and since a locally symmetric Finsler metric is reversible, the isometry $I_{x}$ has the property $I_{x}(\tilde{\gamma}(t))=\tilde{\gamma}(-t)$ for all $t \in\left[-r_{0}-\delta, r_{0}+\delta\right]$. This gives us a contradiction with (4) that proves the Claim.

Let us now show that the metrics $g_{F}$ and $F$ are affinely equivalent as in [10, page 74], that is, for every arc length parameterized $F$-geodesic $\tilde{\gamma}$ there exists a nonzero constant $c$ such that $\tilde{\gamma}(c \cdot t)$ is an arc length parameterized $g_{F}$-geodesic. We have already seen that for a short $F$-geodesic segment, the $g_{F}$-geodesic segment with same endpoints has also the same midpoint. Let us repeat the exact argument. Fix a sufficiently small $g_{F}$-geodesically convex set $W \subset M$ and take a $F$-geodesic $\tilde{\gamma}:[-\widetilde{\varepsilon}, \widetilde{\varepsilon}] \rightarrow W$. Let $\gamma:[-\varepsilon, \varepsilon] \rightarrow W$ be the unique shortest $g_{F}$-geodesic such that $\gamma(-\varepsilon)=\tilde{\gamma}(-\widetilde{\varepsilon})$ and $\gamma(\varepsilon)=\tilde{\gamma}(\widetilde{\varepsilon})$. We assume that both geodesics are parameterized by their arc length in the metric $F$ and $g_{F}$ respectively. Let $x=\tilde{\gamma}(0)$ be the midpoint of $\tilde{\gamma}$ and let $I_{x}$ be the $g_{F}$ reflection centered at $x$. Using the previously proved claim, we find that

$$
I_{x}(\gamma(-\varepsilon))=I_{x}(\tilde{\gamma}(-\widetilde{\varepsilon}))=\tilde{\gamma}(\widetilde{\varepsilon})=\gamma(\varepsilon)
$$

and likewise $I_{x}(\gamma(-\varepsilon))=\gamma(\varepsilon)$. By convexity of $W$, we must have $I_{x}(\gamma) \subset W$ and $I_{x}(\gamma(t))=\gamma(-t)$ for all $t \in[-\varepsilon, \varepsilon]$. In particular $I_{x}(\gamma(0))=\gamma(0)$. By uniqueness of the fixed point of $I_{x}$, it follows that $\gamma(0)=x=\tilde{\gamma}(0)$. Thus, for every $F$-geodesic segment $\tilde{\gamma}$ in $W$, its middle point coincides with the middle point of the unique minimal $g_{F}$-geodesic segment with the same ends.

Replacing the geodesic segment $\tilde{\gamma}_{\mid[-\widetilde{\varepsilon}, \widetilde{\varepsilon}]}$ by $\tilde{\gamma}_{\mid[-\widetilde{\varepsilon}, 0]}$ or by $\tilde{\gamma}_{\mid[0, \tilde{\varepsilon}]}$, we also have that $\gamma\left(-\frac{1}{2} \varepsilon\right)=\tilde{\gamma}\left(-\frac{1}{2} \widetilde{\varepsilon}\right)$ and $\gamma\left(\frac{1}{2} \varepsilon\right)=\tilde{\gamma}\left(\frac{1}{2} \widetilde{\varepsilon}\right)$. Iterating this procedure, we obtain that $\gamma(s \cdot \varepsilon)=\tilde{\gamma}(s \cdot \widetilde{\varepsilon})$ for all $s$ in a dense subset of $[-1,1]$, this implies the geodesic segments $\gamma$ and $\tilde{\gamma}$ coincide after the affine reparameterization $t \mapsto(\widetilde{\varepsilon} / \varepsilon) t$. We obtain locally symmetric space in our definition is also locally symmetric in the definitions in $[4 ; 7]$. 
that $F$ is Berwald whose associated connection is the Levi-Civita connection of $g_{F}$; see [10, page 74]. Thus, Theorem 9.2 is proved for strongly convex Finsler metrics.

In order to complete the proof for an arbitrary Finsler metrics $F$, we consider the Finsler metric $F_{\alpha}$ given by

$$
F_{\alpha}(\xi)=\sqrt{F(\xi)^{2}+\alpha \cdot g_{F}(\xi, \xi)}
$$

where $\alpha>0$ is some parameter. The metric $F_{\alpha}$ is $C^{2}-$ smooth and strictly convex. The reflections $I_{x}$ are evidently isometries of $F_{\alpha}$, so that $F_{\alpha}$ is locally symmetric. We then just proved that $F_{\alpha}$ is Berwald and its associated connection is the LeviCivita connection of $g_{F_{\alpha}}$. Since the reflections $I_{x}$ are evidently isometries of $g_{F_{\alpha}}$, the metrics $g_{F_{\alpha}}$ is affinely equivalent to $g_{F}$ for any $\alpha>0$. Then, for every $\alpha>0$, the function $F_{\alpha}$ is preserved by the parallel transport of the Levi-Civita connection of $g_{F}$. It follows that $F=\lim _{\alpha \rightarrow 0} F_{\alpha}$ is also preserved by the parallel transport of the Levi-Civita connection of $g_{F}$ implying it is Berwald as we claimed.

Corollary 9.4 Every locally symmetric $C^{2}$-smooth Finsler manifold is locally isometric to a globally symmetric Finsler space.

Proof We consider the Binet-Legendre metric $g_{F}$ of our locally symmetric Finsler space $(M, F)$. Since $\left(M, g_{F}\right)$ is also locally symmetric, by the classical results of Cartan [20, theorem 5.1], it is locally isometric to a simply connected globally symmetric Riemannian space $(\bar{M}, g)$. We identify a small open set $U \subset M$ with an open neighborhood set $V \subset \bar{M}$. This defines a Finsler metric $\bar{F}$ on $V$. Now extend the Finsler metric $\bar{F}$ to the whole of $\bar{M}$ using the procedure in (E) of the remark in Section 8, with the help of parallel transport of the Levi-Civita connection of $g$. Since the metric is Berwald and the manifold is simply connected, we obtain a well defined Finsler metric on $\bar{M}$. This metric (we denote it by $\bar{F}$ ) is evidently locally symmetric. Since $g$ and its isometries are real-analytic, the metric $\bar{F}$ is globally symmetric as we claimed.

Remark 9.5 Corollary 9.4 gives us a local description of locally symmetric $\left(C^{2}-\right.$ smooth) Finsler spaces (in special cases this description was obtained in $[14 ; 16]$, and by Planche in [38; 39]). Indeed, take a globally symmetric simply connected Riemannian space $(M, g)$ and consider the isometry subgroup $G$ generated by all reflections. The group $G$ acts transitively on $M$. At one point $x \in M$, consider a smooth Minkowski norm $F_{x}: T_{x} M \rightarrow \mathbb{R}$ such that it is invariant with respect to the stabilizer $G_{x}$ of the point $x$. Next, extend $F_{x}$ to all points with the help of the action of $G$, ie, for an isometry $g \in G$ with $g(x)=y$ put $F_{y}\left(d_{x} g(\xi)\right)=F_{x}(\xi)$. By Corollary 9.4, any 
$C^{2}$-smooth locally symmetric Finsler space is locally isometric to one constructed by this procedure.

\section{The Minkowski functionals and other conformal invari- ants of a Finsler manifold}

The Minkowski functionals are a family of $(n+1)$ invariants associated to a bounded convex set $\Omega$ lying in an $n$-dimensional Euclidean vector space $\left(E^{n}, g\right)$. The standard way to define them is via the Steiner Formula

$$
\operatorname{Vol}^{n}\left(\Omega+t \mathbb{B}^{n}\right)=\sum_{j=0}^{n}\left(\begin{array}{l}
n \\
j
\end{array}\right) \mathrm{W}_{j}^{n}(\Omega) t^{j},
$$

where $\mathbb{B}^{n} \subset E^{n}$ is the Euclidean unit ball. Since the tangent space $T_{x} M$ of a Finsler manifold $(M, F)$ is an Euclidean space (with scalar product given by the BinetLegendre metric $g_{F}$ ), the Minkowski functionals of the $F$-unit ball $\Omega_{x} \subset T_{x} M$ are well defined. We have thus defined on the Finsler manifold $(M, F)$ a family of $n+1$ functions

$$
w_{k}^{n}: M \rightarrow \mathbb{R}, \quad k=0,1, \ldots, n,
$$

(the function $w_{n}^{n}$ is in fact a constant, it is the volume of the Euclidean unit ball). Observe that by construction, these functions are invariant under a conformal deformation of the Finsler metric. It is not difficult to check that if the Finsler metric $F$ is $C^{k}$-partially smooth, then the Minkowski functionals $w_{k}^{n}$ are $C^{k}$-smooth functions on $M$.

Let us construct two additional conformal invariants: At every point $x$ one sets

$$
\mathcal{M}(x)=\max _{0 \neq \xi \in T_{x} M} \frac{F(x, \xi)}{\sqrt{g(\xi, \xi)}}, \quad \mu(x)=\min _{0 \neq \xi \in T_{x} M} \frac{F(x, \xi)}{\sqrt{g(\xi, \xi)}} .
$$

It is easy to show that the functions $\mathcal{M}$ and $\mu$ are continuous, but even if the Finsler metric is smooth, these functions may be nonsmooth.

The invariants defined in the previous subsection can be used in addressing the following.

Equivalence problem for Finsler metrics Let $F_{1}$ and $F_{2}$ be Finsler metrics defined on the discs $U_{1}$ and $U_{2}$. Decide if $\left(U_{1}, F_{1}\right)$ is conformally equivalent to $\left(U_{2}, F_{2}\right)$, in the sense that there exists a diffeomorphism $f: U_{1} \rightarrow U_{2}$ that sends the metric $F_{1}$ to the metric $\lambda \cdot F_{2}$ for a certain function $\lambda$ on $U_{2}$. 
One may also consider the similar isometric equivalence problem. This one has been addressed by Chern in his 1948 paper [9], where he solved it by tensorial methods. His method only works for smooth and strongly convex Finsler metrics.

For the conformal equivalence problem, we propose the following test, which only gives a necessary condition, but which works without smoothness assumptions and is quite stable and manageable from a computational viewpoint. Consider the mappings $\Phi_{i}: U_{i} \rightarrow \mathbb{R}^{n+2}, i=1,2$, given by

$$
\Phi_{i}(x)=\left(w_{0}^{n}(x), \ldots, w_{n-1}^{n}(x), \mu(x), \mathcal{M}(x)\right) .
$$

If the Finsler metrics are conformally equivalent, the images of these mappings (which are in general $n$-dimensional objects in $\mathbb{R}^{n+2}$ ) coincide. Thus, if there exists at least one point that belongs to the first image and not the second, then the metrics are not conformally equivalent.

Note that the test may fail in some instances. In particular this test can never distinguish between two Riemannian metrics and it is in fact quite delicate to decide whether two Riemannian metrics $g_{1}$ and $g_{2}$ are locally isometric or conformally equivalent.

\section{Appendix: Elementary properties of the Binet-Legendre met- ric}

Let $V$ be an $n$-dimensional real vector space and $F: V \rightarrow \mathbb{R}$ be a Minkowski norm on $V$. One defines a scalar product $g_{F}^{*}$ on the dual space $V^{*}$ by the formula

$$
g_{F}^{*}(\theta, \varphi)=\frac{(n+2)}{\lambda(\Omega)} \int_{\Omega}(\theta(\eta) \cdot \varphi(\eta)) d \lambda(\eta)
$$

where $\Omega=\{\xi \in V \mid F(\xi)<1\}$ is the unit sphere associated to $F$ and $\lambda$ is a Lebesgue measure on $V$. The Binet-Legendre metric on $V$ is the scalar product $g_{F}$ on $V$ dual to $g_{F}^{*}$.

Proposition 12.1 The transformation $F \mapsto g_{F}$ satisfies the following properties:

(a) If $F$ is Euclidean, ie $F(\xi)=\sqrt{g(\xi, \xi)}$ for some scalar product $g$, then $g_{F}=g$.

(b) If $A \in \mathrm{GL}(V)$, then $g_{A^{*} F}=A^{*} g_{F}$.

(c) $g_{\kappa F}=\kappa^{2} g_{F}$ for any $\kappa>0$.

(d) If $\frac{1}{c} \cdot F_{1} \leq F_{2} \leq c \cdot F_{1}$ for some constant $c>0$, then

$$
\frac{1}{c^{2 n}} \cdot g_{F_{1}} \leq g_{F_{2}} \leq c^{2 n} \cdot g_{F_{1}} \text {. }
$$


Proof We first prove (a). Suppose $F=\sqrt{g}$ is Euclidean and let $e_{1}, e_{2}, \ldots, e_{n}$ be an orthonormal basis on $(V, g)$ and $x_{1}, x_{2}, \ldots, x_{n}$ be the corresponding coordinate system. The convex set $\Omega$ coincides with the unit ball $\Omega=\mathbb{B}^{n}=\left\{x \in V \mid \sum x_{i}^{2}<1\right\}$ and formula (6) gives

$$
g_{F}^{*}\left(\varepsilon_{i}, \varepsilon_{i}\right)=\frac{(n+2)}{\operatorname{Vol}\left(\mathbb{B}^{n}\right)} \int_{\mathbb{B}^{n}} x_{i}^{2} d x
$$

where $\varepsilon_{i}=e_{i}^{b}$. Now the integral on the left hand side computes as

$$
\int_{\mathbb{B}^{n}} x_{i}^{2} d x=\frac{1}{n} \int_{\mathbb{B}^{n}} \sum_{i=1}^{n} x_{i}^{2} d x=\frac{1}{n} \int_{S^{n-1}} \int_{0}^{1} r^{n+1} d r d \sigma=\frac{\operatorname{Area}\left(S^{n-1}\right)}{n(n+2)} .
$$

$\operatorname{But} \operatorname{Area}\left(S^{n-1}\right)=n \cdot \operatorname{Vol}\left(\mathbb{B}^{n}\right)$ and we thus have

$$
g_{F}^{*}\left(\varepsilon_{i}, \varepsilon_{i}\right)=\frac{(n+2)}{\operatorname{Vol}\left(\mathbb{B}^{n}\right)} \cdot \frac{\operatorname{Area}\left(S^{n-1}\right)}{n(n+2)}=1 .
$$

If $j \neq i$, then

$$
g_{F}^{*}\left(\varepsilon_{i}, \varepsilon_{j}\right)=\frac{(n+2)}{\operatorname{Vol}\left(\mathbb{B}^{n}\right)} \int_{\mathbb{B}^{n}} x_{i} x_{j} d x=0,
$$

because the function $x_{i} x_{j}$ is antisymmetric with respect to the orthogonal transformation $x_{i} \mapsto-x_{i}$. It follows that $\varepsilon_{1}, \ldots, \varepsilon_{n}$ is an orthonormal basis of $V^{*}$ for the scalar product $g_{F}^{*}$. By duality, $e_{1}, \ldots, e_{n}$ is also an orthonormal basis of $V$ for the scalar product $g_{F}$ and therefore $g_{F}=g$.

We now prove property (b). If $A \in \mathrm{GL}(V)$, then the unit ball $\Omega_{A}$ associated to $A^{*} F=A \circ F$ is the set $A^{-1} \cdot \Omega$, indeed

$$
\Omega_{A}=\{\xi \in V \mid F(A \xi)<1\}=\left\{A^{-1} \eta \in V \mid F(\eta)<1\right\}=A^{-1} \cdot \Omega .
$$

Therefore

$$
\begin{aligned}
g_{A^{*} F}^{*}(\theta, \theta) & =\frac{(n+2)}{\lambda\left(A^{-1} \Omega\right)} \int_{A^{-1} \Omega} \theta(\eta)^{2} d \lambda(\eta) \\
& =\frac{(n+2)}{\left|\operatorname{det}\left(A^{-1}\right)\right| \cdot \lambda(\Omega)} \int_{A^{-1} \Omega} \theta(\eta)^{2} d \lambda(\eta) .
\end{aligned}
$$

Setting $\xi=A \eta$, we have from the change of variable formula

$$
\int_{A^{-1}}(\Omega) \theta(\eta)^{2} d \lambda(\eta)=\int_{\Omega} \theta\left(A^{-1} \xi\right)^{2}\left|\operatorname{det}\left(A^{-1}\right)\right| d \lambda(\xi),
$$


and thus

$$
g_{A^{*} F}^{*}(\theta, \theta)=\frac{(n+2)}{\lambda(\Omega)} \int_{\Omega} \theta\left(A^{-1} \xi\right)^{2} d \lambda(\xi)=g_{F}^{*}\left(\theta \circ A^{-1}, \theta \circ A^{-1}\right) .
$$

This is the relation between $g_{A^{*} F}^{*}$ and $g_{F}^{*}$. In the space $V$, we then have by duality

$$
g_{A^{*} F}(\xi, \xi)=g_{F}(A \xi, A \xi)
$$

Property (c) is the special case of property (b) corresponding to scalar matrices.

To prove (d), let $F_{1}, F_{2}$ be two Minkowski norms satisfying $\frac{1}{c} \cdot F_{1} \leq F_{2} \leq c \cdot F_{1}$. Then the corresponding unit balls also satisfy

$$
\frac{1}{c} \cdot \Omega_{1} \subset \Omega_{2} \subset c \cdot \Omega_{1} .
$$

This implies in particular that

$$
\frac{1}{\lambda\left(\Omega_{2}\right)} \leq \frac{c^{n}}{\lambda\left(\Omega_{1}\right)}
$$

We also have

$$
\int_{\Omega_{2}} \theta(\eta)^{2} d \lambda(\eta) \leq \int_{c \cdot \Omega_{1}} \theta(\eta)^{2} d \lambda(\eta)=c^{n} \cdot \int_{\Omega_{1}} \theta(\xi)^{2} d \lambda(\xi)
$$

(set $\xi=c \eta$ ). Therefore

$$
\frac{(n+2)}{\lambda\left(\Omega_{2}\right)} \int_{\Omega_{2}} \theta(\eta)^{2} d \lambda(\eta) \leq c^{2 n} \cdot \frac{(n+2)}{\lambda\left(\Omega_{1}\right)} \int_{\Omega_{1}} \theta(\eta)^{2} d \lambda(\eta),
$$

that is,

$$
g_{F_{2}}^{*}(\theta, \theta) \leq c^{2 n} \cdot g_{F_{1}}^{*}(\theta, \theta) .
$$

The dual scalar product satisfies

$$
g_{F_{1}}(\xi, \xi) \leq c^{2 n} \cdot g_{F_{2}}(\xi, \xi)
$$

Remark 12.2 Formula (6) associates an ellipsoid in $V^{*}$ (the unit ball of the metric $g_{F}^{*}$ ) to an arbitrary convex body $\Omega \subseteq V$. This ellipsoid is called the Binet ellipsoid of $\Omega$ and appears in classical mechanics. The unit ball $B \subset V$ of the metric $g_{F}$ is the polar dual of the Binet ellipsoid. It is related to another classic object: the Legendre ellipsoid $\mathcal{L}$ of $\Omega$ which is the unique ellipsoid such that

$$
\int_{\mathcal{L}} \theta^{2}(\xi) d \lambda(\xi)=\int_{\Omega} \theta^{2}(\xi) d \lambda(\xi)
$$


for any $\theta \in V^{*}$. The Legendre ellipsoid $\mathcal{L}$ is related to the $g_{F}$-unit ball $B$ by the relation

$$
\mathcal{L}=\left(\frac{\lambda(\Omega)}{\lambda(B)}\right)^{\frac{1}{n+2}} \cdot B,
$$

which can be proved from (2) and Proposition 12.1(a).

The integral (7) is called the moment of inertia of $\Omega$ in the codirection $\theta$. Thus the Legendre ellipsoid is the unique ellipsoid having the same moment of inertia as $\Omega$ in all possible codirections and it has the following mechanical interpretation: The motion of a homogenous rigid body $\Omega$ which freely moves in 3-space around the point 0 and is subjected to no external force is dynamically equivalent to a similar motion of its Legendre ellipsoid; see Legendre [26].

\section{References}

[1] D V Alekseevskiŭ, Groups of conformal transformations of Riemannian spaces, Mat. Sb. 89(131) (1972) 280-296, 356 MR0334077 In Russian, translated in: Math. USSR-Sb 18 (1972), 285-301

[2] D Bao, On two curvature-driven problems in Riemann-Finsler geometry, from: "Finsler geometry, Sapporo 2005-in memory of Makoto Matsumoto", (S V Sabau, H Shimada, editors), Adv. Stud. Pure Math. 48, Math. Soc. Japan, Tokyo (2007) 19-71 MR2389251

[3] D Bao, S-S Chern, Z Shen, An introduction to Riemann-Finsler geometry, Graduate Texts in Mathematics 200, Springer, New York (2000) MR1747675

[4] V N Berestovskiü, Generalized symmetric spaces, Sibirsk. Mat. Zh. 26 (1985) 3-17, 221 MR788325

[5] M Berger, Sur les groupes d'holonomie homogène des variétés à connexion affine et des variétés riemanniennes, Bull. Soc. Math. France 83 (1955) 279-330 MR0079806

[6] H Busemann, The geometry of geodesics, Academic Press, New York (1955) MR0075623

[7] H Busemann, B B Phadke, Two theorems on general symmetric spaces, Pacific J. Math. 92 (1981) 39-48 MR618044

[8] P Centore, Volume forms in Finsler spaces, Houston J. Math. 25 (1999) 625-640 MR1829124

[9] S-S Chern, Local equivalence and Euclidean connections in Finsler spaces, Sci. Rep. Nat. Tsing Hua Univ. Ser. A. 5 (1948) 95-121 MR0031812

[10] S-S Chern, Z Shen, Riemann-Finsler geometry, Nankai Tracts in Mathematics 6, World Scientific Publishing Co. Pte. Ltd., Hackensack, NJ (2005) MR2169595 
[11] G de Rham, Sur la reductibilité d'un espace de Riemann, Comment. Math. Helv. 26 (1952) 328-344 MR0052177

[12] S Deng, Z Hou, The group of isometries of a Finsler space, Pacific J. Math. 207 (2002) 149-155 MR1974469

[13] S Deng, Z Hou, Homogeneous Finsler spaces of negative curvature, J. Geom. Phys. 57 (2007) 657-664 MR2271210

[14] S Deng, Z Hou, On symmetric Finsler spaces, Israel J. Math. 162 (2007) 197-219 MR2365860

[15] J Ferrand, The action of conformal transformations on a Riemannian manifold, Math. Ann. 304 (1996) 277-291 MR1371767

[16] P Foulon, Locally symmetric Finsler spaces in negative curvature, C. R. Acad. Sci. Paris Sér. I Math. 324 (1997) 1127-1132 MR1451935

[17] D Fried, Closed similarity manifolds, Comment. Math. Helv. 55 (1980) 576-582 MR604714

[18] P Hartman, Ordinary differential equations, John Wiley \& Sons, New York (1964) MR0171038

[19] E Heil, D Laugwitz, Finsler spaces with similarity are Minkowski spaces, Tensor 28 (1974) 59-62 MR0355918

[20] S Helgason, Differential geometry, Lie groups, and symmetric spaces, Pure and Applied Mathematics 80, Academic Press [Harcourt Brace Jovanovich Publishers], New York (1978) MR514561

[21] S Ishihara, Homogeneous Riemannian spaces of four dimensions, J. Math. Soc. Japan 7 (1955) 345-370 MR0082717

[22] C-W Kim, Locally symmetric positively curved Finsler spaces, Arch. Math. (Basel) 88 (2007) 378-384 MR2311846

[23] S Kobayashi, T Nagano, Riemannian manifolds with abundant isometries, from: "Differential geometry (in honor of Kentaro Yano)", (M Obata, S Kobayashi, editors), Kinokuniya, Tokyo (1972) 195-219 MR0358623

[24] N H Kuiper, Compact spaces with a local structure determined by the group of similarity transformations in $E^{n}$, Nederl. Akad. Wetensch., Proc. 53 (1950) 1178-1185 MR0039248 In Russian, translated in: Indagationes Math. 12 (1950) 411-418

[25] R S Kulkarni, Conformally flat manifolds, Proc. Nat. Acad. Sci. U.S.A. 69 (1972) 2675-2676 MR0307113

[26] A M Legendre, Traité des fonctions elliptiques et des intégrales eulériennes, Volume 1, Huzard-Courcier (1825)

[27] J Liouville, Extension au cas des trois dimensions de la question du tracé géographique, Applications de l'analyse à la géométrie (1850) 609-617 
[28] J Liouville, Théorème sur l'équation $d x^{2}+d y^{2}+d z^{2}=\lambda\left(d \alpha^{2}+d \beta^{2}+d \gamma^{2}\right)$, J. Math. Pures et Appliquées (1850)

[29] R L Lovas, J Szilasi, Homotheties of Finsler manifolds, SUT J. Math. 46 (2010) 23-34 MR2732642

[30] E Lutwak, D Yang, G Zhang, A new ellipsoid associated with convex bodies, Duke Math. J. 104 (2000) 375-390 MR1781476

[31] E Lutwak, D Yang, G Zhang, $L_{p}$ John ellipsoids, Proc. London Math. Soc. 90 (2005) 497-520 MR2142136

[32] M Matsumoto, Conformally Berwald and conformally flat Finsler spaces, Publ. Math. Debrecen 58 (2001) 275-285 MR1807590

[33] S Matsumoto, Foundations of flat conformal structure, from: "Aspects of lowdimensional manifolds", (Y Matsumoto, S Morita, editors), Adv. Stud. Pure Math. 20, Kinokuniya, Tokyo (1992) 167-261 MR1208312

[34] V S Matveev, Riemannian metrics having common geodesics with Berwald metrics, Publ. Math. Debrecen 74 (2009) 405-416 MR2521384

[35] V S Matveev, H-B Rademacher, M Troyanov, A Zeghib, Finsler conformal Lichnerowicz-Obata conjecture, Ann. Inst. Fourier (Grenoble) 59 (2009) 937-949 MR2543657

[36] V D Milman, A Pajor, Isotropic position and inertia ellipsoids and zonoids of the unit ball of a normed $n$-dimensional space, from: "Geometric aspects of functional analysis (1987-88)", (J Lindenstrauss, V D Milman, editors), Lecture Notes in Math. 1376, Springer, Berlin (1989) 64-104 MR1008717

[37] D Montgomery, H Samelson, Transformation groups of spheres, Ann. of Math. 44 (1943) 454-470 MR0008817

[38] P Planche, Géométrie de Finsler sur les espaces symétriques, Thèse Genève (1995)

[39] P Planche, Structures de Finsler invariantes sur les espaces symétriques, C. R. Acad. Sci. Paris Sér. I Math. 321 (1995) 1455-1458 MR1366100

[40] R Schoen, On the conformal and CR automorphism groups, Geom. Funct. Anal. 5 (1995) 464-481 MR1334876

[41] R Schoen, S-T Yau, Conformally flat manifolds, Kleinian groups and scalar curvature, Invent. Math. 92 (1988) 47-71 MR931204

[42] J Simons, On the transitivity of holonomy systems, Ann. of Math. 76 (1962) 213-234 MR0148010

[43] Z I Szabó, Berwald metrics constructed by Chevalley's polynomials arXiv: math/0601522

[44] Z I Szabó, Positive definite Berwald spaces, Structure theorems on Berwald spaces, Tensor 35 (1981) 25-39 MR614132 
[45] I Vaisman, C Reischer, Local similarity manifolds, Ann. Mat. Pura Appl. 135 (1983) 279-291 MR750537

[46] C Vincze, A new proof of Szabó's theorem on the Riemann-metrizability of Berwald manifolds, Acta Math. Acad. Paedagog. Nyházi. 21 (2005) 199-204 MR2162616

[47] H-C Wang, On Finsler spaces with completely integrable equations of Killing, J. London Math. Soc. 22 (1947) 5-9 MR0022431

[48] K Yano, On n-dimensional Riemannian spaces admitting a group of motions of order $n(n-1) / 2+1$, Trans. Amer. Math. Soc. 74 (1953) 260-279 MR0052860

Mathematisches Institut, Friedrich-Schiller Universität Jena

Ernst-Abbe-Platz 2, D-07737 Jena, Germany

Section de Mathématiques, École Polytechnique Féderale de Lausanne

Station 8, CH-1015 Lausanne, Switzerland

vladimir.matveev@uni-jena.de, marc.troyanov@epfl.ch

http://users.minet.uni-jena.de/ matveev/,

http://personnes.epfl.ch/marc.troyanov

Proposed: Dmitri Burago

Seconded: Yasha Eliashberg, Jean-Pierre Otal
Received: 19 January 2012

Revised: 15 May 2012 